TRANSACTIONS OF THE

AMERICAN MATHEMATICAL SOCIETY

Volume 302, Number 2, August 1987

\title{
PRIME IDEALS IN ENVELOPING RINGS
}

\author{
D. S. PASSMAN
}

\begin{abstract}
Let $L$ be a Lie algebra over the field $K$ of characteristic 0 and let $U(L)$ denote its universal enveloping algebra. If $R$ is a $K$-algebra and $L$ acts on $R$ as derivations, then there is a natural ring generated by $R$ and $U(L)$ which is denoted by $R \# U(L)$ and called the smash product of $R$ by $U(L)$. The aim of this paper is to describe the prime ideals of this algebra when it is Noetherian. Specifically we show that there exists a twisted enveloping algebra $U(X)$ on which $L$ acts and a precisely defined one-to-one correspondence between the primes $P$ of $R \# U(L)$ with $P \cap R=0$ and the $L$-stable primes of $U(X)$. Here $X$ is a Lie algebra over some field $C \supseteq K$.
\end{abstract}

Let $L$ be a Lie algebra over the field $K$ and let $U(L)$ denote its universal enveloping algebra. If $R$ is a $K$-algebra and $L$ acts on $R$ as derivations, then this action determines in a natural manner a ring generated by $R$ and $U(L)$ which is denoted by $R \# U(L)$ and called the smash product of $R$ by $U(L)$. The aim of this paper is to study the prime ideals $P$ of $R \# U(L)$ disjoint from $R$, that is with $P \cap R=0$. The main result here describes these primes when char $K=0$ and $R \# U(L)$ is right Noetherian.

When dealing with smash products, it is frequently necessary to study more general objects. For example if $X$ is a Lie ideal of $L$, then $R \# U(L)$ is determined by its subalgebra $R \# U(X)$ and the quotient $U(L / X)$. Indeed

$$
R \# U(L)=(R \# U(X)) \# U(L / X)
$$

provided that the last \# is interpreted as a twisted smash product. This is necessary since the extension $X \triangleleft L$ need not be split. In addition, if $R$ is a prime ring and $S$ is its symmetric ring of quotients, then we have to extend $R \# U(L)$ to $S \# U(L)$. This in itself causes no difficulties until we try to replace the ground field $K$ by $F=\mathbf{Z}(S)$. The problem here is that $L$ can act nontrivially on $F$ so that $F L \subseteq S \# U(L)$ is not necessarily the $F$-Lie algebra $F \otimes_{K} L$. At this point one wonders what sort of object we are actually looking at.

Because of these questions, we begin this paper by discussing this more general object which we might call a universal enveloping ring. Roughly speaking we are given $V \supseteq R \supseteq K$ where $K$ is a field, $R$ is a $K$-algebra and $V$ is a left $K$-vector space. In addition, $V$ is a Lie ring with both $R$ and $K$ as Lie ideals and with the obvious sorts of relations. We denote by $U=U(V, R, K)$ the universal ring containing this structure. Notice that if $R \# U(L)$ is given as above, then this ring is $U(V, R, K)$ where $V=R \oplus L$. Furthermore, the extension to $S$ and to $F=\mathbf{Z}(S)$ then becomes $U\left(V^{\prime}, S, F\right)$ where $V^{\prime}=S+F V$. This is all described in the first

Received by the editors January 31, 1986.

1980 Mathematics Subject Classification (1985 Revision). Primary 16A12, 17 B35.

Research supported in part by N.S.F. Grant No. MCS 82-19678. 
section except for the tedious proof of the Poincaré-Birkhoff-Witt theorem which is relegated to the last section.

With this mechanism is place, we begin our study of the prime ideals of $U=$ $U(V, R, K)$ under the assumption that $R$ is prime and char $K=0$. We start by extending the ring to $U^{\prime}=U\left(V^{\prime}, S, F\right)$ where $S=Q_{s}(R)$ is the symmetric Martindale ring of quotients of $R, F=\mathbf{Z}(S)$ is the extended centroid of $R$ and $V^{\prime}=S+F V$. If $W=\mathbf{C}_{V^{\prime}}(S)$ is the centralizer of $S$ in $V^{\prime}$, then $W$ is an $F$-Lie subalgebra of $V^{\prime}$ and we show that

$$
T=\mathbf{C}_{U^{\prime}}(S)=U(W, F, F) .
$$

In other words, $T$ is generated by its elements of degree $\leq 1$ and it is in fact a twisted enveloping algebra over $F$ of the Lie algebra $W / F$. Thus $T$ is a well-understood subring of $U^{\prime}$. Furthermore, $V$ acts on $T$ as derivations.

We now make the additional assumption that $R$ is right Noetherian and $\operatorname{dim}_{K} V / R<\infty$. Then $W$ as above is finite dimensional over $F$ and we show that there is a precisely prescribed one-to-one correspondence between the prime ideals $P$ of $U$ with $P \cap R=0$ and the $V$-stable prime ideals $\tilde{P}$ of $T=U(W, F, F)$. Indeed $P=\tilde{P} U^{\prime} \cap U$. Since $\operatorname{dim}_{F} W / F \leq \operatorname{dim}_{K} V / R$ this then yields a number of corollaries on incomparability and prime length. Furthermore, except in the totally $\mathrm{X}$-inner case where $V^{\prime}=S+W$, we conclude that $U / P$ is a semiprimitive ring. We remark that the Noetherian hypothesis is used at precisely one point at the end of proof of the one-to-one correspondence. Possibly it is not required, but we do not see an alternate argument at this time.

This paper was motivated by a desire to extend the crossed product techniques of [6] to enveloping algebras. A result of this nature already appears in [2] where one-dimensional Lie algebras are studied over more general and more approprate coefficient rings. A second motivation was the desire to better understand the results of [1] and in so doing we have borrowed a number of its techniques. It is clear that much of the material here carries over to enveloping rings of restricted Lie algebras in characteristic $p>0$. Furthermore analogous ideas yield results on Noetherian crossed products. These will be considered elsewhere.

Finally I would like to thank J. Bergen and S. Montgomery for visiting me in Madison during the fall semester of 1985 and for many stimulating conversations. They rekindled my enthusiasm for this problem and convinced me that the Noetherian case might be of interest.

1. Universal enveloping rings. We are mainly concerned with the smash product $R \# U(L)$. However to study these inductively and to make necessary field extensions, we must deal with a somewhat more general object. In this section we define this more general construction, mention some concrete examples, state the appropriate Poincaré-Birkhoff-Witt theorem and obtain some useful corollaries.

Let $K$ be a field and let $V$ be a left $K$-vector space which is a Lie ring under the operation [ , ]. We suppose that $V$ contains $R$, an associative $K$-algebra with 1 and hence that $V \supseteq R \supseteq K$. Assume that:

(L1) $[R, V] \subseteq R$ and $[K, V] \subseteq K$.

(L2) For each $v \in V$ the map $\delta_{v}: r \rightarrow[r, v]$ is a derivation on $R$.

(L3) For each $v, w \in V, a, b \in K$ and $r, s \in R$

$$
[a v, b w]=a b[v, w]+a[v, b] w-b[w, a] v, \quad[r, s]=r s-s r .
$$


In particular $R$ and $K$ are Lie ideals of $V$ and [ , ] restricted to $R$ is the usual Lie product determined by the ring. Note also that any derivation of a ring stabilizes its center. Thus if $K=\mathbf{Z}(R)$ then (L2) implies that $[K, V] \subseteq K$. The motivation for (L2) and (L3) above is

LEMMA 1.1. Let $T$ be any ring with usual Lie product [ , ] and let $T \supseteq V$. Suppose that $V \supseteq R \supseteq K$ as above, $V$ is a left $K$-vector space and that $[V, V] \subseteq$ $V,[R, V] \subseteq R$ and $[K, V] \subseteq K$. Then (L2) and (L3) are satisfied.

ProOF. It is well known that if $t \in T$ then $\operatorname{ad}_{t}$ is a derivation on $T$. For (L3) observe that

$$
\begin{aligned}
{[a v, b w] } & =a v b w-b w a v \\
& =a(b v+[v, b]) w-b(a w+[w, a]) v \\
& =a b[v, w]+a[v, b] w-b[w, a] v .
\end{aligned}
$$

We remark that for this structure it is necessary to assume that $K$ is central in $R$. Indeed let $T$ be as above with $T \supseteq V \supseteq R \supseteq K$ but do not assume that $K$ is central in $R$. If $v \in V \backslash R$ then

$$
[a v, r]=a[v, r]+[a, r] v .
$$

Thus from $[V, R] \subseteq R$ we must have $[K, R]=0$.

We are concerned with the structure of the largest ring $T$, as in Lemma 1.1, which is generated by $V$. A priori, we do not know that such a ring exists. That is part of the content of the PBW theorem which also yields an $R$-basis for the ring. Formally we say that $U=U(V, R, K)$ is a universal enveloping ring for $V \supseteq R \supseteq K$ if:

(U1) $U$ is an associative ring generated by $V$ with identity element $1 \in R$.

(U2) [ , ] on $V$ is the restriction of the usual Lie product on the ring $U$.

(U3) Let $W \supseteq S \supseteq K$ be any triple of left $K$-vector spaces where $W$ is a Lie ring and $S$ is a $K$-algebra. Assume that ${ }^{-}: V \rightarrow W$ is a $K$-linear Lie homomorphism whose restriction to $R$ is a $K$-algebra homomorphism ${ }^{-}: R \rightarrow S$. If $T$ is a ring generated by $W$ such that [ , ] on $W$ is the restriction of the usual Lie product on $T$, then there exists a unique ring homomorphism $U \rightarrow T$ which extends the map ${ }^{-}$on $V$.

Properties (U1)-(U3) imply immediately that $U$ if it exists is unique up to $V$ isomorphism.

Assume that $V$ is contained in a ring $T$ and let $x_{1}, x_{2}, \ldots$ be a well-ordered sequence of elements of $V$. Then a monic monomial in the $x$ 's is a product of the form $\mu=x_{i_{1}} x_{i_{2}} \cdots x_{i_{n}}$ with $i_{1} \leq i_{2} \leq \cdots \leq i_{n}$.

LEMMA 1.2. Let $T \supseteq V \supseteq R \supseteq K$ be as in the preceding lemma and suppose in addition that $T$ is generated as a ring by $V$. Let $x_{1}, x_{2}, \ldots$ be a well-ordered $K$-basis for a complement for $R$ in $V$. Then the monic monomials in the $x$ 's span $T$ as a left $R$-module.

PROOF. Let $\tau_{n}$ denote the linear span of all products of $r$ 's and $x$ 's involving at most $n x$-factors. We note that $\left[x_{i}, x_{j}\right] \in \tau_{1}$ and $\left[x_{i}, r\right] \in \tau_{0}$. The formulas in (L3) imply that if $\alpha$ is a generating product in $\tau_{n}$ and if $\alpha^{\prime}$ is obtained from $\alpha$ 
by interchanging two adjacent factors at least one of which is an $x$, then $\alpha \equiv \alpha^{\prime}$ $\bmod \tau_{n-1}$. It follows that $\alpha \equiv r \mu \bmod \tau_{n-1}$ for some $r \in R$ and monic monomial $\mu$. Now proceed by induction on $n$.

The following result asserts that $U(V, R, K)$ exists. We prove it in $\S 5$.

THEOREM 1.3 (PBW). Let $V \supseteq R \supseteq K$ be given satisfying (L1)-(L3) and let $x_{1}, x_{2}, \ldots$ be a well-ordered $K$-basis for a complement for $R$ in $V$. Then $U=$ $U(V, R, K)$ exists and is a free left $R$-module with basis consisting of all monic monomials in the $x$ 's.

Let us consider some examples with $[K, V]=0$ so that $U$ is a $K$-algebra. First $U(V, K, K)$ is just a twisted enveloping algebra of the Lie algebra $V / K$. Furthermore if the extension $K \triangleleft V$ is split, that is if $V=K \oplus L$ with $L$ a $K$-subspace and $[L, L] \subseteq L$, then $U(V, K, K)=U(L)$ is an ordinary enveloping algebra. In the same way, $U(V, R, K)$ is a twisted smash product $R \# U(V / R)$ and if $V=R \oplus L$ with $[L, L] \subseteq L$, then $U(V, R, K)$ is the ordinary smash product $R \# U(L)$. Finally if $\operatorname{dim}_{K} V / R=1$ and say $V=R+K x$, then $U(V, R, K)$ is the Ore extension $R\left[x ; \delta_{x}\right]$ where $\delta_{x}$ is the derivation $x$ induces on $R$.

The PBW theorem has numerous corollaries. We list several which we will need.

COROLlaRY 1.4. Let $V \supseteq R \supseteq K$ be given satisfying (L1)-(L3) and assume that $T$ is a ring which contains and is generated by $V$. Suppose further that [ , ] on $V$ is the restriction of the usual Lie product on $T$ and let $x_{1}, x_{2}, \ldots$ be a well-ordered $K$-basis for a complement for $R$ in $V$. Then $T i s V$-isomorphic to $U(V, R, K)$ if and only if the monic monomials in the $x$ 's contained in $T$ are (left) $R$-linearly independent.

PROOF. If $T \simeq U$ then the monic monomials are $R$-linearly independent. Conversely if the latter occurs, then by Lemma 1.2 and the universal property (U3), the $V$-homomorphism $U \rightarrow T$ is an isomorphism.

COROLLARY 1.5. Let $V \supseteq R \supseteq K$ be given satisfying (L1)-(L3) and assume that $W \supseteq R$ is a $K$-subspace of $V$ and a Lie subring. Then the subring of $U=$ $U(V, R, K)$ generated by $W$ is isomorphic to $U^{\prime}=U(W, R, K)$ and $U^{\prime} \cap V=W$. Furthermore if $W \triangleleft V$ and $W \neq V$, then $U^{\prime}$ is a $K$-algeba and

$$
U(V, R, K)=U\left(U^{\prime}+V, U^{\prime}, K\right) .
$$

ProOF. It is clear that $W \supseteq R \supseteq K$ also satisfies (L1)-(L3). Let $x_{1}, x_{2}, \ldots$ be a well-ordered $K$-basis for a complement for $R$ in $W$ and let $y_{1}, y_{2}, \ldots$ be a well-ordered $K$-basis for a complement for $W$ in $V$. Then $\left\{x_{i}\right\} \cup\left\{y_{j}\right\}$ is a basis for a complement for $R$ in $V$ and we will order it by insisting that $x_{i}<y_{j}$ for all $i, j$. If $T$ is the subring of $U=U(V, R, K)$ generated by $W$, then $T$ is a left $R$-module spanned by the monic monomials in the $x$ 's. Since the latter are $R$-independent by the PBW theorem, we have $T=U(W, R, K)$ by Corollary 1.4.

Assume in addition that $W \triangleleft V$ and $W \neq V$. If $w \in W, a \in K$ and $v \in V \backslash W$ then

$$
[w, a] v=[w, a v]-a[w, v] \in W
$$

and $[w, a]=0$. Hence $[W, K]=0$ and then $T$ is a $K$-algebra. Note that $[W, V] \subseteq W$ implies that $[T, V] \subseteq T$ and therefore that $T+V$ is a Lie subring of $U$. Thus $T+V \supseteq T \supseteq K$ is also a triple satisfying (L1)-(L3). Furthermore $T \cap V=W$ by 
the PBW theorem and hence $y_{1}, y_{2}, \ldots$ is also a $K$-basis for a complement for $T$ in $T+V$. Since $U$ is generated by $T+V$ and since the monic monomials in the $y$ 's are linearly independent over $T$, by Theorem 1.3 again, we conclude from Corollary 1.4 that $U=U(T+V, T, K)$.

The following result allows us to extend the base $\operatorname{ring} R$ via localization.

COROLLARY 1.6. Let $V \supseteq R \supseteq K$ be given satisfying (L1)-(L3) and let $S$ be an overring of $R$. Suppose that for each $v \in V$ the derivation $\delta_{v}: R \rightarrow R$ extends to a unique derivation on $S$. Then $S$ is a $K$-algebra and $U=U(V, R, K)$ extends uniquely to $U^{\prime}=U\left(V^{\prime}, S, K\right)$ where $V^{\prime}=V+S$ and $V \cap S=R$. Furthermore $U^{\prime}=S U$.

ProOF. If $k \in K$, then $\delta_{k}=0$ on $R$ extends by uniqueness to $\delta_{k}=0$ on $S$. Thus $S$ is a $K$-algebra. Again the hypothesis on extension and uniqueness implies that the Lie antihomomorphism $\delta: V \rightarrow \operatorname{Der}(R)$ extends to a Lie antihomomorphism $\delta: V \rightarrow \operatorname{Der}(S)$. Now form the $K$-vector space direct sum $W=S \oplus V$ and define [ , ] on $W$ linearly to extend the given operation on $V$ and the usual Lie bracket on the ring $S$. Furthermore $-[v, s]=[s, v]=\delta_{v}(s)$ for $v \in V$ and $s \in S$.

$W$ is easily seen to be a Lie ring. Indeed only the Jacobi identity need be verified and by linearity we can assume that the elements to be tested are each contained in $S$ or $V$. If all three are in $S$ or $V$ the result is obvious. If two are in $V$ then the identity follows from the fact that $\delta$ is a Lie antihomomorphism. Finally if $s, t \in S$ and $v \in V$ then since $\delta_{v}$ is a derivation

$$
\delta_{v}([s, t])=\left[\delta_{v}(s), t\right]+\left[s, \delta_{v}(t)\right]
$$

and this yields the remaining case of the Jacobi identity.

Next it is easy to see that

$$
I=\{(r,-r) \in S \oplus V \mid r \in R\}
$$

is a Lie ideal of $W$. In fact $[S, I]=0$ and for $v \in V$

$$
[(r,-r), v]=\left(\delta_{v}(r),-\delta_{v}(r)\right) .
$$

Now we can set $V^{\prime}=W / I$. Then via the Lie homomorphism $W \rightarrow V^{\prime}$ both $S$ and $V$ embed isomorphically and using the same symbols to denote their images we have $V^{\prime}=S+V$ and $S \cap V=R$.

It remains to show that the triple $V^{\prime} \supseteq S \supseteq K$ satisfies (L1)-(L3). Since (L1) and (L2) are clear, we need only verify the first part of (L3). Furthermore that part also holds if $v, w \in V$ or $v, w \in S$. Thus by linearity and $V^{\prime}=S+V$, it suffices to consider $v \in V$ and $w=s \in S$. Notice first that if $b=1$ and $w=r \in R$ then (L3) for $V \supseteq R \supseteq K$ yields $[a v, r]=a[v, r]$. Thus by uniqueness of extension we have

$$
[a v, b s]=a[v, b s]=a b[v, s]+a[v, b] s
$$

and this yields (L3) in this final case since $[s, a]=0$.

We conclude from the PBW theorem that $U^{\prime}=U\left(V^{\prime}, S, K\right)$ exists and we let $U$ be the subring of $U^{\prime}$ generated by $V$. If $x_{1}, x_{2}, \ldots$ is a well-ordered $K$-basis for a complement for $R$ in $V$, then from $V^{\prime}=S+V$ we see that $x_{1}, x_{2}, \ldots$ is also a basis for a complement for $S$ in $V^{\prime}$. We now know that the monic monomials in the $x$ 's are a left $S$-basis for $U^{\prime}$. Thus $U^{\prime}=S U$ and since these monomials are also left $R$-independent, Corollary 1.4 yields $U=U(V, R, K)$. 
The next result handles the sort of field extensions we require in a rather trivial manner.

COROLlary 1.7. Let $U=U(V, R, K)$ be given and let $F \supseteq K$ be a central subfield of $R$ with $[F, V] \subseteq F$. Then $U=U(F V, R, F)$ with $F V / R \simeq F \otimes_{K}(V / R)$ as a left $F$-vector space.

PROOF. Let $x_{1}, x_{2}, \ldots$ be a well-ordered $K$-basis for a complement for $R$ in $V$. Since these elements are $R$-linearly independent, by Theorem 1.3, it follows that $\left\{x_{i}\right\}$ is also an $F$-basis for a complement for $R$ in $F V \subseteq U$. Furthermore $F V$ is a Lie subring of $U,[F, V] \subseteq F$ and $F \subseteq \mathbf{Z}(R)$, so it follows easily from Lemma 1.1 that $F V \supseteq R \supseteq F$ satisfies (L1)-(L3). We conclude from Corollary 1.4 that $U=U(F V, R, F)$.

We remark that if $F=\mathbf{Z}(R)$ in the above, then the hypothesis $[F, V] \subseteq F$ is automatic since $V$ acts as derivations on $R$. If $I$ is an ideal of $R$ then $I$ is $V$-stable if $[I, V] \subseteq I$. The following comes as no surprise.

COROLlary 1.8. Let $U=U(V, R, K)$ be given and let $I \neq R$ be a $V$-stable ideal of $R$. Then $I U \triangleleft U$ and

$$
U / I U \simeq U(V / I, R / I, K)
$$

ProOF. Since $I$ is a Lie ideal of $V$ and an ideal of $R$, it follows immediately that $V / I \supseteq R / I \supseteq K$ also satisfies (L1)-(L3). Thus by (U3) the map $V \rightarrow V / I$ extends to a ring epimorphism $U(V, R, K) \rightarrow U(V / I, R / I, K)$. Furthermore if $x_{1}, x_{2}, \ldots$ is a well-ordered $K$-basis for a complement for $R$ in $V$, then $\left\{x_{i}+I\right\}$ is a $K$-basis for a complement for $R / I$ in $V / I$. With this we conclude immediately from Theorem 1.3 that the kernel of this ring homomorphism is precisely $I U$.

Of course many other properties of smash products also carry over to enveloping rings. For example $U=U(V, R, K)$ is a filtered ring, filtered by the left $R$ submodules $U_{n}=V^{n}$. Furthermore it follows from the PBW theorem that the associated graded ring

$$
\bar{U}=\oplus \sum_{n=0}^{\infty} \frac{U_{n}}{U_{n-1}}
$$

where $U_{-1}=0$ is isomorphic to the polynomial ring $R\left[x_{1}, x_{2}, \ldots\right]$. In particular if $R$ is right or left Noetherian and if $\operatorname{dim}_{K} V / R<\infty$, then $\bar{U}$ is also right or left Noetherian. Thus (see [5, p. 165]) we have

THEOREM 1.9. Let $U=U(V, R, K)$ be given and assume that $\operatorname{dim}_{K} V / R<\infty$. If $R$ is right or left Noetherian, then so is $U$.

2. Centralizer of the base ring. In this section we begin our work on the structure of prime ideals in enveloping rings. If $U=U(V, R, K)$ is given, we determine the structure of $\mathbf{C}_{U}(R)$ in case char $K=0$ and $\mathbf{Z}(R)=K$. The characteristic 0 assumption is definitely needed here, but the other hypothesis can frequently be finessed. As will be apparent, it is necessary to study a slightly more general situation. Namely we assume that the base $\operatorname{ring} R$ is of the form $S \otimes_{K} T$ and we compute $\mathbf{C}_{U}(S)$. 
To be more precise we let $F$ be a field and we let $S$ and $T$ be $F$-algebras. The conditions we require are:

(C1) $V \supseteq S \otimes_{F} T \supseteq F$ satisfies (L1)-(L3).

(C2) $F=\mathbf{Z}(S)$ and $\operatorname{char} F=0$.

(C3) There exists a left $F$-subspace $X$ of $V$ with $V=S \otimes T+X,[S, X] \subseteq S$ and $[T, X] \subseteq T$.

We of course view $S$ and $T$ as $F$-subalgebras of $S \otimes T$. Indeed, in this paper, $S \otimes T$ frequently means $S T$ in some larger ring with $S$ and $T$ commuting and appropriately disjoint.

PROPOSITION 2.1. Let $V \supseteq S \otimes_{F} T \supseteq F$ satisfy (C1)-(C3) and set $U=$ $U\left(V, S \otimes_{F} T, F\right)$. If $W=\mathbf{C}_{V}(S)$, then $W \supseteq T$ is an $F$-Lie subalgebra of $V, W \cap$ $(S \otimes T)=T$ and $\mathbf{C}_{U}(S)=U(W, T, F)$.

PROOF. If $\left\{t_{i}\right\}$ is an $F$-basis for $T$, then every element $\alpha$ of $S \otimes T$ is uniquely of the form

$$
\alpha=\sum_{i} s_{i} \otimes t_{i}=\sum_{i} s_{i} t_{i}
$$

In particular if $\alpha \in \mathbf{C}_{S \otimes T}(S)$, then for all $r \in S$

$$
0=\alpha r-r \alpha=\sum_{i}\left(s_{i} r-r s_{i}\right) t_{i}
$$

so each $s_{i} \in \mathbf{Z}(S)=F \subseteq T$. Thus $\alpha=\sum s_{i} t_{i} \in T$ and we conclude that $T=$ $\mathrm{C}_{S \otimes T}(S)$.

Since $S$ is a Lie subring of $V$ it follows that $W=\mathbf{C}_{V}(S)$ is also a Lie subring. Since $S$ and $W$ commute with $F, W$ is an $F$-Lie algebra. Furthermore

$$
W \cap(S \otimes T)=\mathbf{C}_{S \otimes T}(S)=T
$$

so $T$ is a Lie ideal of $W$.

Let $w_{1}, w_{2}, \ldots$ be a well-ordered $F$-basis for a complement for $T$ in $W$. Since $W \cap(S \otimes T)=T$, these elements $w_{i}$ are also $F$-linearly independent modulo $S \otimes T$. Thus since $V=S \otimes T+X$ by (C3), we can extend $\left\{w_{i}\right\}$ to obtain a basis for a complement for $S \otimes T$ in $V$ be adding $x_{1}, x_{2}, \ldots$ with all $x_{i} \in X$. We assume that $\left\{x_{i}\right\}$ is well-ordered and that the $x$ 's come before the $w$ 's. It follows from the PBW theorem that every element $\alpha$ of $U$ is uniquely of the form

$$
\alpha=\sum_{i, \sigma, \tau} s_{i \sigma \tau} t_{i} \sigma \tau
$$

where $\left\{t_{i}\right\}$ is the basis for $T$ mentioned above, $\sigma$ is a monic monomial in the $x$ 's and $\tau$ is a monic monomial in the $w$ 's. Of course $s_{i \sigma \tau} \in S$. Note that $t_{i}$ commutes with $S$ so we can write $\alpha$ uniquely as

$$
\alpha=\sum_{i, \tau} t_{i} \alpha_{i \tau} \tau
$$

where $\alpha_{i \tau}=\sum_{\sigma} s_{i \sigma \tau} \sigma$. The goal is to show that $\alpha \in \mathbf{C}_{U}(S)$ if and only if each $\alpha_{i \tau} \in F$.

Note that $T$ and each $\tau$ centralizes $S$. Thus if each $\alpha_{i \tau} \in F \subseteq T$ then clearly $\alpha$ centralizes $S$. Conversely suppose that $\alpha \in \mathbf{C}_{U}(S)$. Then for all $r \in S$

$$
0=\alpha r-r \alpha=\sum_{i, \tau} t_{i}\left(\alpha_{i \tau} r-r \alpha_{i \tau}\right) \tau .
$$


Now $\alpha_{i \tau}$ is a sum of monomials of the form $s x_{j_{1}} x_{j_{2}} \cdots x_{j_{a}}$ with $j_{1} \leq j_{2} \leq \cdots \leq$ $j_{a}$. Furthermore in computing $\left(s x_{j_{1}} x_{j_{2}} \cdots x_{j_{a}}\right) r-r\left(s x_{j_{1}} x_{j_{2}} \cdots x_{j_{a}}\right)$ we need only interchange $x$ 's with elements of $S$. Indeed the $x$ 's remain in their natural order even though some may be deleted in the process. It follows from $[S, X] \subseteq S$ that $\alpha_{i \tau} r-r \alpha_{i \tau}$ is also a sum of monomials of the form $s x_{j_{1}} x_{j_{2}} \cdots x_{j_{a}}$ and uniqueness of expression now implies that $\alpha_{i \tau} r=r \alpha_{i \tau}$ for all $r \in S$. Thus each $\alpha_{i \tau} \in \mathbf{C}_{U}(S)$.

Fix $i, \tau$ and write

$$
\beta=\alpha_{i \tau}=\sum_{\sigma} s_{\sigma} \sigma
$$

where the $\sigma$ 's are monic monomials in the $x$ 's and say $\operatorname{deg} \beta=n \geq 0$. If $r \in S$ then $0=\beta r-r \beta$. We first consider terms in $\beta r-r \beta$ of degree $n$. Since $x_{i}$ commutes with $r$ modulo terms of lower degree, it follows that the degree $n$ terms are precisely

$$
0=\sum_{\operatorname{deg} \sigma=n}\left[s_{\sigma}, r\right] \sigma .
$$

Hence we deduce that if $\operatorname{deg} \sigma=n$ then $s_{\sigma}$ centralizes $S$ and we have $s_{\sigma} \in \mathbf{Z}(S)=F$. If $n=0$ we conclude that $\beta \in F$ as required. Thus suppose by way of contradiction that $n>0$.

We now consider the degree $n-1$ terms in $\beta r-r \beta$ and we may suppose that all monomials in $\beta$ involve $x_{1}, x_{2}, \ldots, x_{j}$ only. First suppose $\operatorname{deg} \sigma=n$ and write

$$
\sigma=x_{1}^{a_{1}} x_{2}^{a_{2}} \cdots x_{j}^{a_{j}} .
$$

Since $s_{\sigma} \in F$ and since $S$ commutes with the $x$ 's modulo lower degree terms, it follows that the degree $n-1$ contribution of $s_{\sigma} \sigma$ in $\beta r-r \beta$ is

$$
s_{\sigma} \sum_{k=1}^{j} a_{k}\left[x_{k}, r\right] x_{1}^{a_{1}} \cdots x_{k}^{a_{k}-1} \cdots x_{j}^{a_{j}} .
$$

On the other hand, if $\operatorname{deg} \sigma=n-1$ then its degree $n-1$ contribution in $\beta r-r \beta$ is $\left[s_{\sigma}, r\right] \sigma$. We conclude therefore that

$$
0=\sum_{\operatorname{deg} \sigma=n} s_{\sigma} \sum_{k=1}^{j} a_{k}\left[x_{k}, r\right] x_{1}^{a_{1}} \cdots x_{k}^{a_{k}-1} \cdots x_{j}^{a_{j}}+\sum_{\operatorname{deg} \sigma=n-1}\left[s_{\sigma}, r\right] \sigma .
$$

Fix a monomial $\eta=x_{1}^{b_{1}} x_{2}^{b_{2}} \cdots x_{j}^{b_{j}}$ of degree $n-1$ which comes from some $\sigma$ of degree $n$ in $\beta$ by lowering one of the exponents by one. Then the coefficient of $\eta$ in the above is precisely

$$
0=\sum_{k=1}^{j} f_{k}\left(b_{k}+1\right)\left[x_{k}, r\right]+[s, r] .
$$

Here $f_{k} \in F$ is the coefficient of the $\sigma$-term of the degree $n$ obtained from $\eta$ by increasing the $x_{k}$ exponent to $b_{k}+1$. By assumption some $f_{k}$ is not zero. Also $s \in S$ is the coefficient of the monic monomial $\eta$ of degree $n-1$ in $\beta$. Since $[F, S]=0$ it follows that

$$
\sum_{k=1}^{j} f_{k}\left(b_{k}+1\right) x_{k}+s \in \mathbf{C}_{V}(S)=W
$$


Notice that all $b_{k}+1$ are (nonzero) positive integers, $F$ is a field of characteristic 0 and some $f_{k}$ is nonzero. Thus the above implies that $x_{1}, x_{2}, \ldots, x_{j}$ are $F$-linearly dependent modulo $W+S \otimes T$, a contradiction.

We conclude that $\mathbf{C}_{U}(S)$ consists of all elements of the form $\sum_{i, \tau} t_{i} f_{i \tau} \tau=\sum_{\tau} t_{\tau} \tau$ where $f_{i \tau} \in F, t_{\tau}=\sum_{i} t_{i} f_{i \tau} \in T$ and $\tau$ is a monic monomial in the $w$ 's. In other words, by the PBW theorem, $\mathbf{C}_{U}(S)=U(W, T, F)$ and the result follows.

We remark that the degree function used above is the usual one obtained from the filtration of $U$. As a consequence of that result we obtain the main theorem of this section which also indicates why the above structure (C1)-(C3) is of interest.

THEOREM 2.2. Let $U=U(V, R, K)$ be an enveloping ring with $\mathbf{Z}(R)=K$ and char $K=0$. If $W=\mathbf{C}_{V}(R)$ then

$$
T=\mathbf{C}_{U}(R)=U(W, K, K)
$$

is a twisted enveloping algebra of $W / K$ over $K$. Furthermore, $W$ is a Lie ideal of $V,[R, V] \subseteq R,[T, V] \subseteq T$ and

$$
U=U\left(R \otimes_{K} T+V, R \otimes_{K} T, K\right) .
$$

Proof. Since $R$ is a Lie ideal of $V$ so is $W=\mathbf{C}_{V}(R)$. Furthermore by Proposition 2.1 with $S=R, T=F=K$ we have $\mathbf{C}_{U}(R)=U(W, K, K)$, a twisted enveloping algebra over $K$. If we now call this centralizer $T$, then $T$ is certainly a $K$-algebra and $[T, V] \subseteq T$.

Note that $R+W \triangleleft V$ so by Corollary 1.5 (or trivially if $R+W=V$ ) we have $U=U\left(U^{\prime}+V, U^{\prime}, K\right)$ where $U^{\prime}=U(R+W, R, K)$. Thus it remains to show $U^{\prime}=R \otimes_{K} T$. Since $R$ and $W$ commute, they generate $U^{\prime}=R T$ and it suffices to show that some $K$-basis for $T$ is $R$-independent. To this end, let $\left\{w_{i}\right\}$ be a $K$-basis for a complement for $K$ in $W$. Then $T=U(W, K, K)$ has as a $K$-basis the monic monomials in the $w$ 's. But $W \cap R=\mathbf{Z}(R)=K$ so $\left\{w_{i}\right\}$ is $K$-linearly independent modulo $R$ and hence it is part of a basis for a complement for $R$ in $V$. It now follows from the PBW theorem again that the monic monomials in the $w$ 's are $R$-independent.

We remark that the above is not true if char $K \neq 0$. For example, let char $K=$ $p>0, R=K_{(\varsigma)}$ and let $U=R[x ; \partial / \partial \zeta]$. Then $\mathbf{C}_{U}(R)=R\left[x^{p}\right]$ is not generated by its elements of degree $\leq 1$. On the other hand, one does expect an analogous result to hold in characteristic $p>0$ for restricted Lie rings and restricted enveloping rings.

We now return to the characteristic 0 situation. Since we will only require the above result when $K=\mathbf{Z}(R)$ we just briefly mention a more general formulation. First if $F=\mathbf{Z}(R)$ is a field, then $[F, V] \subseteq F$ and, by Corollary 1.7, $U=U(F V, R, F)$. Thus $\mathbf{C}_{U}(R)=U(W, F, F)$ where $W=\mathbf{C}_{F V}(R)$. Second, if $Z=\mathbf{Z}(R)$ consists of regular elements of $R$ (for example if $R$ is prime) then we can form the central localization $S=Z^{-1} R$. Since any derivation of $R$ extends uniquely to one of $S$, $U$ embeds in $U^{\prime}=U(S+V, S, K)$ by Corollary 1.6. Since $\mathbf{C}_{U^{\prime}}(R)=\mathbf{C}_{U^{\prime}}(S)$ and since $\mathbf{Z}(S)=Z^{-1} Z$ is a field, an appropriate result exists here.

The following lemma contains a crucial observation.

LEMMA 2.3. Let $V \supseteq S \otimes_{F} T \supseteq F$ satisfy (C1)-(C3) and let $I \neq T$ be an ideal of $T$ such that $S \otimes I$ is a $V$-stable ideal of $S \otimes T$. If ${ }^{-}: U \rightarrow U /(S \otimes I) U$ is 
the natural epimorphism, then $\bar{U}=U\left(\bar{V}, \bar{S} \otimes_{F} \bar{T}, F\right)$ where $\bar{S} \simeq S$ and $\bar{T}=T / I$. Furthermore $\mathbf{C}_{\bar{U}}(\bar{S})=\overline{\mathbf{C}_{U}(S)}$.

Proof. Since $S \otimes I$ is a $V$-stable ideal of $S \otimes T$, it follows from Corollary 1.8 that $(S \otimes I) U \triangleleft U$,

$$
\bar{U}=U /(S \otimes I) U=U(\bar{V}, \overline{S \otimes T}, F)
$$

and

$$
\overline{S \otimes T}=(S \otimes T) /(S \otimes I)=S \otimes(T / I) .
$$

It remains to study the centralizers and certainly $\overline{\mathbf{C}_{U}(S)} \subseteq \mathbf{C}_{\bar{U}}(\bar{S})$.

For the other inclusion let $1=t_{0}, t_{1}, t_{2}, \ldots$ be an $F$-basis for a complement for $I$ in $T$ and let $v \in V$ with $\bar{v} \in \mathbf{C}_{\bar{V}}(\bar{S})$. Since $V=S \otimes T+X$ by (C3) we see that $v$ is congruent modulo $S \otimes I$ to an element of the form

$$
v^{\prime}=\sum_{i} s_{i} t_{i}+x
$$

with $s_{i} \in S$ and $x \in X$. The centralizer property says that for all $r \in S, v^{\prime} r$ $-r v^{\prime} \in(S \otimes I) U$. But observe that $[X, S] \subseteq S$ so

$$
v^{\prime} r-r v^{\prime} \in(S \otimes I) U \cap(S \otimes T)=S \otimes I .
$$

Since $t_{0}=1$ and $[x, r] \in S$ we conclude that for all $r \in S$

$$
\left[s_{0}+x, r\right] t_{0}+\sum_{i \neq 0}\left[s_{i}, r\right] t_{i} \in S \otimes I
$$

so by definition of $\left\{t_{i}\right\}$ we have $\left[s_{0}+x, S\right]=0$ and $\left[s_{i}, S\right]=0$ for $i \neq 0$. In other words,

$$
v^{\prime}=\left(s_{0}+x\right) t_{0}+\sum_{i \neq 0} s_{i} t_{i} \in \mathbf{C}_{V}(S)
$$

and we conclude that $\overline{\mathbf{C}_{V}(S)}=\mathbf{C}_{\bar{V}}(\bar{S})$.

Finally observe that $\bar{V} \supseteq \bar{S} \otimes \bar{T} \supseteq F$ also satisfies (C1)-(C3). Thus by Proposition $2.1, \mathrm{C}_{\bar{U}}(\bar{S})$ is generated as a ring by $\mathbf{C}_{\bar{V}}(\bar{S})$ and hence it is the image of the subring of $U$ generated by $\mathbf{C}_{V}(S)$, namely $\mathbf{C}_{U}(S)$.

The next section will consider the link between the ideals of $U(V, R, K)$ and the centralizer of the base ring.

3. Ideals of the centralizer. To proceed further it is necessary to localize $R$. Let $R$ be a prime ring. Then the left Martindale ring of quotients $Q_{l}(R)$ exists and is uniquely characterized by:

(Q1) $Q_{l}(R) \supseteq R$ with the same 1 .

(Q2) If $q \in Q_{l}$ then there exists $0 \neq A \triangleleft R$ with $A q \subseteq R$.

(Q3) If $q \in Q_{l}, 0 \neq A \triangleleft R$ and $A q=0$, then $q=0$.

(Q4) If $f:{ }_{R} A \rightarrow{ }_{R} R$ is given with $0 \neq A \triangleleft R$, then there exists $q \in Q_{l}$ with $a f=a q$ for all $a \in A$.

An important subring of $Q_{l}$ is

$$
Q_{s}(R)=\left\{q \in Q_{l} \mid \text { there exists } 0 \neq B \triangleleft R \text { with } q B \subseteq R\right\} .
$$

This is the symmetric Martindale ring of quotients which is uniquely characterized via the following symmetric formulation. 
(S1) $Q_{s}(R) \supseteq R$ with the same 1 .

(S2) If $q \in Q_{s}$ then there exist $0 \neq A, B \triangleleft R$ with $A q, q B \subseteq R$.

(S3) If $q \in Q_{s}$ and $0 \neq I \triangleleft R$ then either $I q=0$ or $q I=0$ implies $q=0$.

(S4) Let $f:{ }_{R} A \rightarrow{ }_{R} R$ and $g: B_{R} \rightarrow R_{R}$ be given with $0 \neq A, B \triangleleft R$ and suppose that for all $a \in A, b \in B$ we have $(a f) b=a(g b)$. Then there exists $q \in Q_{s}$ with $a f=a q$ and $g b=q b$ for all $a \in A, b \in B$.

For details see $[\mathbf{7}, \S 1]$.

We will be mainly concerned with $Q_{s}(R)$ but for technical reasons we will also have to consider the larger ring $Q_{l}$. We start with

LEMMA 3.1. Let $R$ be a ring, $\mp$ a family of subsets of $R$ and let $R \subseteq S \subseteq U$. Suppose that for all $s \in S$ there exists $A \in \mathcal{F}$ with $A s \subseteq R$ and that for all $B \in \mathcal{F}$ we have $\underline{r}_{U}(B)=0$. Then $\mathbf{C}_{U}(S)=\mathbf{C}_{U}(R)$. Furthermore if $\delta$ is a derivation of $R$ which extends to a derivation of $S$, then the extension is unique.

Proof. Since $R \subseteq S$ we have $\mathbf{C}_{U}(R) \supseteq \mathbf{C}_{U}(S)$. Conversely let $u \in \mathbf{C}_{U}(R)$ and let $s \in S$. Choose $A \in \mathcal{F}$ with $A s \subseteq R$. Then for all $a \in A$

$$
(a s) u=u(a s)=(u a) s=(a u) s
$$

so $A(s u-u s)=0$. Thus $s u-u s \in \underline{r}_{U}(A)=0$ and $u \in \mathbf{C}_{U}(S)$.

For the second part, it suffices to show that if $\delta$ is a derivation of $S$ with $\delta(R)=0$ then $\delta=0$. Let $s \in S$ and let $A \in \mathcal{F}$ with $A s \subseteq R$. Then $\delta(A)=0$ and $\delta(A s)=0$ yield $A \delta(s)=0$ and hence that $\delta(s) \in \underline{r}_{U}(A)=0$.

LEMMA 3.2. Let $R$ be a prime ring. If $S=Q_{l}(R)$ or $Q_{s}(R)$, then every derivation of $R$ extends uniquely to one of $S$.

PROOF. The uniqueness of extension follows from the above with $U=S$ and with $₹$ the nonzero ideals of $R$. We are concerned here with existence. Let $\delta: R \rightarrow$ $R$ be a derivation and let $S=Q_{l}(R)$. If $s \in S$, choose $0 \neq A \triangleleft R$ with $A s \subseteq R$ and define $f_{s}: A^{2} \rightarrow R$ by $b f_{s}=\delta(b s)-\delta(b) s$. Note that $\delta\left(A^{2}\right) \subseteq A$ so $\delta(b) s \in R$ and $b f_{s} \in R$. Certainly $f_{s}$ is additive and since $\delta$ is a derivation on $R$ we have

$$
(r b) f_{s}=\delta(r b s)-\delta(r b) s=r \delta(b s)+\delta(r) b s-r \delta(b) s-\delta(r) b s=r\left(b f_{s}\right) .
$$

It follows from (Q4) that there exists $q_{s} \in Q_{l}$ with $b f_{s}=b q_{s}$ for all $b \in A^{2}$. Furthermore, in view of (Q2) and this formula, it follows that $q_{s}$ is uniquely determined by $s$ independent of the choice of $A$.

Notice that if $r \in R$, then

$$
b f_{r}=\delta(b r)-\delta(b) r=b \delta(r)
$$

so $q_{r}=\delta(r)$ in this case. We can therefore define $\delta(s)=q_{s}$ for all $s \in S$. By definition of $\delta(s)=q_{s}$ we then have

$$
\delta(b s)=b f_{s}+\delta(b) s=b \delta(s)+\delta(b) s
$$

for all $b \in A^{2}$. It remains to show that $\delta: S \rightarrow S$ is a derivation. To this end let $s, t \in S$ and choose $0 \neq A \triangleleft R$ with $A s, A t \subseteq R$. Since $A^{3} s \subseteq A^{2}$ and $A^{2} s t \subseteq R$ it follows that for all $b \in A^{4}$

$$
\begin{aligned}
b \delta(s t) & =\delta(b s t)-\delta(b) s t=\delta(b s) t+b s \delta(t)-\delta(b) s t \\
& =b \delta(s) t+\delta(b) s t+b s \delta(t)-\delta(b) s t=b(\delta(s) t+s \delta(t))
\end{aligned}
$$

and thus $\delta(s t)=\delta(s) t+s \delta(t)$ by (Q3). 
Finally if $s \in Q_{s}(R) \subseteq S$ then there exists $0 \neq B \triangleleft R$ with $s B \subseteq R$. Then $\delta\left(s B^{2}\right) \subseteq R$ and $s \delta\left(B^{2}\right) \subseteq R$ so $\delta(s) B^{2} \subseteq R$. Thus $\delta(s) \in Q_{s}(R)$ and $\delta$ restricts to a derivation of $Q_{s}$.

Note that the following result does not assume the hypothesis (C2).

LEMMA 3.3. Let $V \supseteq S \otimes_{F} T \supseteq F$ satisfy hypotheses (C1) and (C3) and set $U=U(V, S \otimes T, F)$. Suppose that $R$ is a prime ring, $S=Q_{l}(R)$ and that $X=F Y$ where $Y$ is an additive subset of $X$ satisfying $[R, Y] \subseteq R$. If $I$ is any nonzero $(R, R)$-subbimodule of $U$, then there exist $0 \neq A \triangleleft R$ and $0 \neq \alpha \in \mathbf{C}_{U}(S)$ with $A \alpha \subseteq I$.

PROOF. Note that

$$
V=S \otimes T+X=S \otimes T+F Y
$$

Let $t_{1}, t_{2}, \ldots$ be an $F$-basis for $T$. By the above we can let $y_{1}, y_{2}, \ldots$ be a wellordered $F$-basis for a complement for $S \otimes T$ in $V$ with all $y_{i} \in Y$. Then by the PBW theorem every element $\beta$ of $U$ is uniquely writable as

$$
\beta=\sum_{i, \tau} s_{i \tau} t_{i} \tau
$$

where $\tau$ is a monic monomial in the $y$ 's and $s_{i \tau} \in S$.

Note that if $\beta \neq 0$ is as above then there exists $0 \neq B \triangleleft R$ with $0 \neq B s_{i \tau} \subseteq R$ for all $s_{i \tau} \neq 0$. In other words, $I \cap U_{1} \neq 0$ where $U_{1}=\sum_{i, \tau} R t_{i} \tau$. Now choose an element $0 \neq \beta \in I \cap U_{1}$ subject to two constraints. First, $\beta$ has the minimal degree $n$ of all nonzero elements of $I \cap U_{1}$. Second among the elements of $I \cap U_{1}$ of degree $n, \beta$ has the minimal number of nonzero terms of the form $r_{i \tau} t_{i} \tau$ with $\operatorname{deg} \tau=n$. Let us assume that $\beta$ involves the elements $y_{1}, y_{2}, \ldots, y_{j}$ and $t_{1}, t_{2}, \ldots, t_{k}$ only. For convenience let $\mathcal{M}$ be the set of all monomials of the form $t_{i} \tau$ with $1 \leq i \leq k$ and $\tau$ a monic monomial in $y_{1}, y_{2}, \ldots, y_{j}$ of degree $\leq n$. Furthermore if $\operatorname{deg} \tau=n$ then we only include those $t_{i} \tau$ which occur in the expression for $\beta$. Thus $\mathcal{M}=\left\{\mu_{0}, \mu_{1}, \ldots, \mu_{m}\right\}$ is a finite set, each $\mu_{i}$ has degree $\leq n$ and there is at least one monomial of degree $n$, say $\mu_{0}$.

Set $U_{2}=\sum_{i=0}^{m} R \mu_{i}$. We claim that $U_{2}$ is also an $(R, R)$-subbimodule of $U$. Indeed if $\mu \in \mathcal{M}$ then since $T$ centralizes $R$ and $[R, Y] \subseteq R$ we see that $\mu r=r \mu+, \eta$ where $\eta$ is an $R$-linear sum of terms in $\mathcal{M}$ of degree less than $\operatorname{deg} \mu$. By the choice of $\mathcal{M}, I \cap U_{2}$ is nonzero $(R, R)$-subbimodule of $U$ and we let

$$
A=\left\{a \in R \mid \sum_{i=0}^{m} r_{i} \mu_{i} \in I \cap U_{2} \text { with } a=r_{0}\right\} .
$$

Since $\operatorname{deg} \mu_{0}=n$, it follows from the above formula for $\mu r$ that $A$ is a two-sided ideal of $R$. Furthermore since $\mu_{0}$ occurs in the expression for $\beta \in I \cap U_{2}$ we have $A \neq 0$.

We claim that for each $a \in A$ there exists a unique $\gamma \in I \cap U_{2}$ with $\gamma=a \mu_{0}+\cdots$. Indeed if $\gamma, \gamma^{\prime}$ are two such elements with $\gamma^{\prime}=a \mu_{0}+\cdots$, then $\gamma-\gamma^{\prime} \in I \cap U_{2}$ either has degree $<n$ or it has degree $n$ with less terms of degree $n$ than $\beta$. It follows from the choice of $\beta$ that $\gamma-\gamma^{\prime}=0$. We can write the unique $\gamma$ determined by $a$ 
as

$$
\gamma=\sum_{i=0}^{m}\left(a g_{i}\right) \mu_{i}
$$

where $g_{i}: A \rightarrow R$ is a well-defined function with $a g_{0}=a$. Furthermore since $\gamma=a \mu_{0}+\cdots$ implies that $r \gamma=r a \mu_{0}+\cdots$, it follows that each $g_{i}$ is a left $R$ module map. Since $S=Q_{l}(R)$ we conclude from (Q4) that there exist $s_{i} \in S$ with $s_{0}=1$ and $a g_{i}=a s_{i}$ for all $i$.

Set $\alpha=\sum_{i=0}^{m} s_{i} \mu_{i} \in U$ so that by the above $A \alpha \subseteq I \cap U_{2}$. If $r \in R$ then the formula for $\mu r$ along with $s_{0}=1$ shows that

$$
\alpha r-r \alpha=\sum_{i=0}^{m} q_{i} \mu_{i}
$$

with $q_{i} \in S$ and $q_{0}=0$. Furthermore since $A \alpha \subseteq I \cap U_{2}$ and $A r \subseteq A$ we have $A(\alpha r-r \alpha) \subseteq I \cap U_{2}$ and these elements have their $\mu_{0}$ coefficient equal to zero. Uniqueness implies that $A(\alpha r-r \alpha)=0$ so $A q_{i}=0$ and hence $q_{i}=0$. We conclude that $\alpha \in \mathbf{C}_{U}(R)$. Hence by Lemma 3.1, with $\mathcal{F}$ the family of nonzero ideals of $R$, we have $\alpha \in \mathrm{C}_{U}(S)$. Since $A \alpha \subseteq I$, the lemma is proved.

Now we obtain the analogous result for $S=Q_{s}(R)$. Here we use the $(\mathrm{C} 2)$ hypothesis at least in the proof.

LEMMA 3.4. Let $V \supseteq S \otimes_{F} T \supseteq F$ satisfy hypotheses $(\mathrm{C} 1)-(\mathrm{C} 3)$ and set $U=$ $U(V, S \otimes T, F)$. Suppose that $R$ is a prime ring, $S=Q_{s}(R)$ and that $X=F Y$ where $Y$ is an additive subset of $X$ satisfying $[R, Y] \subseteq R$. If $I$ is any nonzero $(R, R)$-subbimodule of $U$, then there exist $0 \neq A \triangleleft R$ and $0 \neq \alpha \in \mathbf{C}_{U}(S)$ with $A \alpha \subseteq I$.

ProOF. Let $S^{\prime}=Q_{l}(R) \supseteq Q_{s}(R)=S$ and let $\mathcal{F}$ denote the family of nonzero ideals of $R$. Since $\mathbf{Z}\left(S^{\prime}\right)$ centralizes $R$, it follows from the definition of $Q_{s}$ that $\mathbf{Z}\left(S^{\prime}\right) \subseteq S$ and hence that $\mathbf{Z}\left(S^{\prime}\right) \subseteq \mathbf{Z}(S)=F$. On the other hand, by Lemma 3.1, $\mathbf{C}_{S^{\prime}}\left(S^{\prime}\right)=\mathbf{C}_{S^{\prime}}(S) \supseteq F$ so we have the reverse inclusion. Therefore $F=\mathbf{Z}\left(S^{\prime}\right)$.

The first goal is to extend the base ring $S \otimes_{F} T$ to $S^{\prime} \otimes_{F} T$ via Corollary 1.6. To this end, let $\delta_{v}$ denote the derivation induced by $v \in V$ on $S \otimes T$. Suppose first that $y \in Y$ so that $[T, y] \subseteq T$ and $[R, y] \subseteq R$. By Lemma 3.2, the derivation $y$ induces on $R$ has a unique extension to both $S$ and $S^{\prime}$. It therefore follows that $\delta_{y}$ extends to $S^{\prime} \otimes T$ with $\delta_{y}\left(S^{\prime}\right) \subseteq S^{\prime}$. Since $V=S \otimes T+F Y$ we now conclude by linearity that every $\delta_{v}$ extends to a derivation on $S^{\prime} \otimes T$. Furthermore uniqueness of extension follows from Lemma 3.1 with the same $\mathcal{F}$ so Corollary 1.6 applies. We conclude that $U$ extends to $U^{\prime}=U\left(V^{\prime}, S^{\prime} \otimes T, F\right)$ where $V^{\prime}=S^{\prime} \otimes T+V$ and $V \cap\left(S^{\prime} \otimes T\right)=S \otimes T$. Note that $V^{\prime}=S^{\prime} \otimes T+F Y$ and $\left[S^{\prime}, F Y\right] \subseteq S^{\prime}$ so $V^{\prime} \supseteq S^{\prime} \otimes T \supseteq F$ satisfies (C1)-(C3).

Let $t_{0}=1, t_{1}, t_{2}, \ldots$ be an $F$-basis for $T$. The next goal is to show that $\mathbf{C}_{U^{\prime}}\left(S^{\prime}\right) \subseteq \mathbf{C}_{U}(S)$. Suppose first that $v^{\prime} \in \mathbf{C}_{V^{\prime}}\left(S^{\prime}\right)$ and write

$$
v^{\prime}=\sum_{i} s_{i}^{\prime} \otimes t_{i}+x
$$


with $s_{i}^{\prime} \in S^{\prime}$ and $x \in X=F Y$. Since $[x, R] \subseteq S$ we see that for all $r \in R$

$$
\begin{aligned}
& 0=\left[v^{\prime}, r\right]=\sum_{i}\left[s_{i}^{\prime}, r\right] \otimes t_{i}+[x, r] \\
& =\left[s_{0}^{\prime}+x, r\right] \otimes t_{0}+\sum_{i \neq 0}\left[s_{i}^{\prime}, r\right] \otimes t_{i} \in S \otimes T .
\end{aligned}
$$

It follows from uniqueness of expression that $s_{i}^{\prime} \subseteq \mathbf{C}_{S^{\prime}}(R) \subseteq S$ for all $i \neq 0$ and that $s_{0}^{\prime}+x \in \mathbf{C}_{V^{\prime}}(R)$. To handle the latter term observe that $x \in X=F Y$ so say $x \in f_{1} Y+f_{2} Y+\cdots+f_{k} Y$ where $f_{1}, f_{2}, \ldots, f_{k} \in F$. Since $[Y, R] \subseteq R$ and $F$ centralizes $R$ this yields

$$
[x, R] \subseteq f_{1} R+f_{2} R+\cdots+f_{k} R
$$

and hence

$$
\left[s_{0}^{\prime}, R\right]=-[x, R] \subseteq f_{1} R+f_{2} R+\cdots+f_{k} R .
$$

Now $s_{0}^{\prime} \in S^{\prime}$ so there exists $0 \neq B \triangleleft R$ with $B s_{0}^{\prime} \subseteq R$. Furthermore there exists $0 \neq C \triangleleft R$ with $f_{j} C \subseteq R$ for all $j=1,2, \ldots, k$. It follows that

$$
\begin{aligned}
s_{0}^{\prime} B & \subseteq B s_{0}^{\prime}+R f_{1}+R f_{2}+\cdots+R f_{k} \\
& \subseteq R+R f_{1}+R f_{2}+\cdots+R f_{k}
\end{aligned}
$$

so $s_{0}^{\prime} B C \subseteq R$. Therefore $s_{0}^{\prime} \in S$ and we have shown that $v^{\prime} \in V$. In other words, $\mathrm{C}_{V^{\prime}}\left(S^{\prime}\right) \subseteq V$ so surely $\mathbf{C}_{V^{\prime}}\left(S^{\prime}\right) \subseteq \mathbf{C}_{V}(S)$. Finally, by Proposition 2.1 applied to $U^{\prime}=U\left(V^{\prime}, S^{\prime} \otimes T, F\right)$ we see that $\mathbf{C}_{U^{\prime}}\left(S^{\prime}\right)$ is the $F$-algebra generated by $\mathbf{C}_{V^{\prime}}\left(S^{\prime}\right)$ so we conclude that $\mathbf{C}_{U^{\prime}}\left(S^{\prime}\right) \subseteq \mathbf{C}_{U}(S)$.

The result now follows easily. Note that $I$ is a nonzero $(R, R)$-subbimodule of $U^{\prime}$. Thus by Lemma 3.3 there exists $0 \neq A \triangleleft R$ and $0 \neq \alpha \in \mathbf{C}_{U^{\prime}}\left(S^{\prime}\right)$ with $A \alpha \subseteq I$. Since $\alpha \in \mathbf{C}_{U^{\prime}}\left(S^{\prime}\right) \subseteq \mathbf{C}_{U}(S)$, the lemma is proved.

With this lemma in place, we no longer need to consider the left Martindale ring of quotients. The above motivates the following structure.

Let $U=U(V, R, K)$ be a universal enveloping ring with $R$ prime and char $K=0$. Let $S=Q_{s}(R)$ and let $F=\mathbf{Z}(S)$. Then $F$ is called the extended centroid of $R$ and it is a field containing $K$. Since every derivation of $R$ uniquely extends to one of $S$, by Lemma 3.2, Corollaries 1.6 and 1.7 imply that $U$ extends to $U^{\prime}=U\left(V^{\prime}, S, F\right)$ where $V^{\prime}=S+F V$. Set $W=\mathrm{C}_{V^{\prime}}(S)$ and $T=\mathrm{C}_{U^{\prime}}(S)$. Then by Theorem 2.2, $\left[T, V^{\prime}\right] \subseteq T$ and $T=U(W, F, F)$ is a twisted enveloping algebra over $F$. For each $(U, U)$-subbimodule $I$ of $U^{\prime}$ we define

$$
\tilde{I}=\{\alpha \in T \mid A \alpha \subseteq I \text { for some } 0 \neq A \triangleleft R\} .
$$

LEMMA 3.5. With the above notation, $\tilde{I}$ is a $V$-stable ideal of $T$. Furthermore for each $\beta \in \tilde{I} U^{\prime}$ there exists $0 \neq B \triangleleft R$ with $B \beta \subseteq I$.

Proof. If $\alpha, \beta \in \tilde{I}$ with $A \alpha, B \beta \subseteq I$, then $(A \cap B)(\alpha+\beta) \subseteq I$ so $\alpha+\beta \in \tilde{I}$. Now let $\gamma \in T$ and choose $0 \neq C \triangleleft R$ with $C \gamma \subseteq U$. Since $\alpha$ and $\gamma$ centralize $R$ we have

$$
A C \alpha \gamma=(A \alpha)(C \gamma) \subseteq I U=I
$$

and

$$
C A \gamma \alpha=(C \gamma)(A \alpha) \subseteq U I=I
$$

so $\alpha \gamma, \gamma \alpha \in \tilde{I}$. Thus $\tilde{I} \triangleleft T$. 
Note that $I, R$ and $T$ are all $V$-stable so $\tilde{I}$ is $V$-stable. Indeed let $\alpha \in \tilde{I}$ and let $\delta_{v}$ be the derivation induced by $v \in V$. If $A \alpha \subseteq I$, then $\delta_{v}\left(A^{2} \alpha\right) \subseteq I$ and $\delta_{v}\left(A^{2}\right) \alpha \subseteq A \alpha \subseteq I$ so $A^{2} \delta_{v}(\alpha) \subseteq I$. Finally if $\beta \in \tilde{I} U^{\prime}$ then $\beta=\sum_{1}^{n} \alpha_{i} \gamma_{i}$ with $\alpha_{i} \in \tilde{I}$ and $\gamma_{i} \in U^{\prime}$. Choose $0 \neq A, C \triangleleft R$ with $A \alpha_{i} \subseteq I$ and $C \gamma_{i} \subseteq U$ for all $i=1,2, \ldots, n$. Then

$$
A C \beta \subseteq \sum_{1}^{n} A C \alpha_{i} \gamma_{i}=\sum_{1}^{n}\left(A \alpha_{i}\right)\left(C \gamma_{i}\right) \subseteq I U=I
$$

and the lemma is proved.

We are concerned with the relationship between $I$ and $\tilde{I}$. The following is the main result of this section.

Proposition 3.6. Let I be a $(U, U)$-subbimodule of $U^{\prime}$. Then with the above notation, $\tilde{I} U^{\prime} \triangleleft U^{\prime}$ and $I \subseteq \tilde{I} U^{\prime}$.

PROOF. We may clearly assume that $1 \notin \tilde{I}$ since otherwise the result is obvious. We apply Theorem 2.2 to $U^{\prime}$ and write it as

$$
U^{\prime}=U\left(S \otimes_{F} T+V^{\prime}, S \otimes T, F\right)
$$

and set

$$
V^{\prime \prime}=S \otimes_{F} T+V^{\prime}=S \otimes_{F} T+F V .
$$

Since both $S$ and $T$ are $V^{\prime}$-stable we see that $V^{\prime \prime} \supseteq S \otimes T \supseteq F$ satisfies (C1)-(C3) with $X=V^{\prime}$.

By Lemma 3.5, $\tilde{I}$ is a $V$-stable ideal of $T$ and thus $S \otimes \tilde{I}$ is an ideal of $S \otimes T$ which is clearly $V^{\prime \prime}$-stable. Therefore Lemma 2.3 applies and we use its notation. In particular Lemma 2.3 asserts that

$$
\overline{U^{\prime}}=U^{\prime} /(S \otimes \tilde{I}) U^{\prime}=U\left(\overline{V^{\prime \prime}}, \bar{S} \otimes \bar{T}, F\right)
$$

where $\bar{T}=T / \tilde{I}$ and $\bar{S} \simeq S$. Furthermore since $T=\mathrm{C}_{U^{\prime}}(S)$,

$$
\mathrm{C}_{\overline{U^{\prime}}}(\bar{S})=\overline{\mathbf{C}_{U^{\prime}}(S)}=\bar{T} \text {. }
$$

Note that

$$
(S \otimes \tilde{I}) U^{\prime}=\tilde{I} S U^{\prime}=\tilde{I} U^{\prime} .
$$

Suppose that $I \nsubseteq \tilde{I} U^{\prime}$. Then $\bar{I}$ is a nonzero $(\bar{R}, \bar{R})$-submodule of $\overline{U^{\prime}}$. Note that

$$
\overline{V^{\prime \prime}}=\bar{S} \otimes \bar{T}+F \bar{V}
$$

with $[\bar{R}, \bar{V}] \subseteq \bar{R},[\bar{S}, F \bar{V}] \subseteq \bar{S}$ and $[\bar{T}, F \bar{V}] \subseteq \bar{T}$. Thus since $\bar{S} \simeq S$ the hypotheses of Lemma 3.4 are satisfied in $\overline{U^{\prime}}$. We conclude that there exist $0 \neq \bar{A} \triangleleft \bar{R}$ and $0 \neq \bar{\alpha} \in \mathbf{C}_{\overline{U^{\prime}}}(\bar{S})$ with $\bar{A} \bar{\alpha} \subseteq \bar{I}$. As we observed above, $\bar{T}=\mathbf{C}_{\overline{U^{\prime}}}(\bar{S})$ so we may assume that $\alpha \in T$. Thus if $A \triangleleft R$ corresponds to $\bar{A}$, then in $U^{\prime}$ we have

$$
A \alpha \subseteq I+(S \otimes \tilde{I}) U^{\prime}=I+\tilde{I} U^{\prime} .
$$

Choose $a \in A \backslash 0$. Since $a \alpha \in I+\tilde{I} U^{\prime}$, it follows from Lemma 3.5 that there exists $0 \neq B \triangleleft R$ with $B a \alpha \subseteq I$. But $\alpha$ commutes with $R$ so this yields

$$
(B a R) \alpha=B a \alpha R \subseteq I R=I .
$$

Since $R$ is prime, $0 \neq B a R \triangleleft R$ so by definition of $\tilde{I}$ we have $\alpha \in \tilde{I}$. Hence $\bar{\alpha}=0$, a contradiction. Thus we must have $\bar{I}=0$ and therefore $I \subseteq \tilde{I} U^{\prime}$.

This of course applies when $I$ is an ideal of $U$ or of $U^{\prime}$. 
4. The prime correspondence. We continue with the notation at the end of the preceding section. In particular, $R$ is a prime $K$-algebra with char $K=0$, $S=Q_{s}(R)$ and $U=U(V, R, K)$ is extended to $U^{\prime}=U\left(V^{\prime}, S, F\right)$ where $F=\mathbf{Z}(S)$ and $V^{\prime}=S+F V$. Furthermore $T=\mathrm{C}_{U^{\prime}}(S)=U(W, F, F)$. Our goal is to link the prime ideals of $T$ to those of $U$ which are disjoint from $R$. With little additional work, we can add to the link the primes of $U^{\prime}$ which are disjoint from $S$.

Observe that $V$ acts as derivations on $T$. If $Q \neq T$ is a $V$-stable ideal of $T$ then $Q$ is said to be $V$-prime if for all $V$-stable ideals $I, J$ of $T$, the inclusion $I J \subseteq Q$ implies $I \subseteq Q$ or $J \subseteq Q$. We begin with

LEMMA 4.1. Let $Q$ be a $V$-stable ideal of $T$ and set $P=Q U^{\prime} \cap U, P^{\prime}=Q U^{\prime}$. Then $P \triangleleft U$ and $P^{\prime} \triangleleft U^{\prime}$ with $P \cap R=P^{\prime} \cap S=0$ and $\tilde{P}=\tilde{P}^{\prime}=Q$. Furthermore if $Q$ is $V$-prime, then $P$ is a prime ideal of $U$ and $P^{\prime}$ is a prime of $U^{\prime}$.

ProOF. We apply Theorem 2.2 to $U^{\prime}$ and write it as

$$
U^{\prime}=U\left(V^{\prime \prime}, S \otimes T, F\right)
$$

where

$$
V^{\prime \prime}=S \otimes_{F} T+V^{\prime}=S \otimes T+F V .
$$

Since $Q$ is a $V$-stable ideal of $T$, it follows that $S \otimes Q$ is an ideal of $S \otimes T$ which is clearly $V^{\prime \prime}$-stable. Thus Lemma 2.3 asserts that $P^{\prime}=Q U^{\prime} \triangleleft U^{\prime}$ and $P^{\prime} \cap(S \otimes T)=$ $S \otimes Q$. Thus $P=P^{\prime} \cap U \triangleleft U$ and $P \cap(S \otimes T) \subseteq S \otimes Q$. It follows that $P^{\prime} \cap S=P \cap R=0$.

Let $\alpha \in \tilde{P}$ or $\tilde{P}^{\prime}$. Then by definition there exists $0 \neq A \triangleleft R$ with

$$
A \alpha \subseteq P^{\prime} \cap(S \otimes T)=S \otimes Q
$$

and hence $\alpha \in Q$. Conversely if $\beta \in Q$ then there exists $0 \neq B \triangleleft R$ with $B \beta \subseteq U$ and then

$$
B \beta=\beta B \subseteq Q U^{\prime} \cap U=P \subseteq P^{\prime} .
$$

We conclude therefore that $\tilde{P}=\tilde{P}^{\prime}=Q$.

Finally assume that $Q$ is $V$-prime. We show that $P$ is prime, the proof for $P^{\prime}$ being identical. Let $I, J \triangleleft U$ with $I J \subseteq P$ and suppose $\alpha \in \tilde{I}, \beta \in \tilde{J}$. If $0 \neq A, B \triangleleft R$ with $A \alpha \subseteq I$ and $B \beta \subseteq J$ then

$$
A B \alpha \beta=(A \alpha)(B \beta) \subseteq I J \subseteq P
$$

so $\alpha \beta \in \tilde{P}$. In other words, $\tilde{I} \tilde{J} \subseteq \tilde{P}=Q$. But $\tilde{I}$ and $\tilde{J}$ are $V$-stable ideals of $T$, by Lemma 3.5, and $Q$ is $V$-prime. It follows that one of $\tilde{I}$ or $\tilde{J}$ is contained in $Q$, say $\tilde{I} \subseteq Q$. We conclude from Proposition 3.6 that

$$
I \subseteq \tilde{I} U^{\prime} \cap U \subseteq Q U^{\prime} \cap U=P
$$

and the lemma is proved.

This seems to be as far as we go in our proof without a Noetherian hypothesis. It is not certain however whether the latter is an essential ingredient in the theorem. In some sense it is used to compensate for the fact that the ideals $A$ of $R$ we have considered are not necessarily $V$-stable. Note that if $T$ is Noetherian, then any $V$-prime ideal of $T$ is necessarily prime by [4, Corollary 1.4] since char $F=0$. This explains why the following lemma is phrased in terms of prime ideals of $T$. We now obtain the reverse direction of the correspondence. 
LEMMA 4.2. Assume in addition that $R$ is right Noetherian and $\operatorname{dim}_{K} V / R<$ $\infty$. Then $U=U(V, R, K)$ is right Noetherian, $\operatorname{dim}_{F} W<\infty$ and $T=U(W, F, F)$ is right and left Noetherian. Furthermore let $P$ be a prime ideal of $U$ with $P \cap R=0$ or let $P^{\prime}$ be a prime ideal of $U^{\prime}$ with $P^{\prime} \cap S=0$. Then $\tilde{P}$ and $\tilde{P}^{\prime}$ are $V$-stable prime ideals of $T$ with $P=\tilde{P} U^{\prime} \cap U, P^{\prime}=\tilde{P}^{\prime} U^{\prime}$.

Proof. $U$ is Noetherian by Theorem 1.9. Furthermore since $W \cap S=F$ and $\operatorname{dim}_{K} V / R<\infty$, it follows that $\operatorname{dim}_{F} W<\infty$. Thus by Theorem 1.9 again, $T=U(W, F, F)$ is both right and left Noetherian.

We first show that $P=\tilde{P} U^{\prime} \cap U$. To this end set $H=\tilde{P} U^{\prime} \cap U$ so that $H \triangleleft U$ and $H \supseteq P$ by Proposition 3.6. Since $U$ is right Noetherian, we can write $H=\sum_{1}^{n} \alpha_{i} U$ and since $\alpha_{i} \in H \subseteq \tilde{P} U^{\prime}$ it follows from Lemma 3.5 that there exists $0 \neq A_{i} \triangleleft R$ with $A_{i} \alpha_{i} \subseteq P$. Letting $A=\bigcap_{1}^{n} A_{i}$ we have $0 \neq A \triangleleft R$ and $A H \subseteq P$. Now $P$ is a prime ideal of $U$ and $H \triangleleft U$ so this implies that either $A \subseteq P$ or $H \subseteq P$. But by assumption $P \cap R=0$ so the first possibility cannot hold and we conclude that $H=P$ as required.

The argument for $P^{\prime}$ is just slightly different since we are not given that $S$ is right Noetherian. Set $H^{\prime}=\tilde{P}^{\prime} U^{\prime}$ so tht $H^{\prime} \triangleleft U^{\prime}$ and $H^{\prime} \supseteq P^{\prime}$. Since $T$ is right Noetherian, $\tilde{P}^{\prime}$ is a finitely generated right ideal of $T$ and hence $H^{\prime}$ is a finitely generated right ideal of $U^{\prime}$. Now proceed as above.

Finally we show that $\tilde{P}$ and $\tilde{P}^{\prime}$ are $V$-prime. Since the proofs are identical in the two cases, we will only consider $\tilde{P}$. Let $I, J$ be $V$-stable ideals of $T$ with $I J \subseteq \tilde{P}$. Since $I U^{\prime}$ and $J U^{\prime}$ are ideals of $U^{\prime}$, by Lemma 4.1, we have $U^{\prime} J \subseteq J U^{\prime}$ and hence

$$
\left(I U^{\prime}\right)\left(J V^{\prime}\right) \subseteq I J U^{\prime} \subseteq \tilde{P} U^{\prime} .
$$

It follows that

$$
\left(I U^{\prime} \cap U\right)\left(J U^{\prime} \cap U\right) \subseteq \tilde{P} U^{\prime} \cap U=P
$$

and thus since $P$ is prime one of these factors is in $P$, say $I U^{\prime} \cap U \subseteq P$. By Lemma 4.1 again this yields

$$
I=\left(I U^{\prime} \cap U\right)^{\sim} \subseteq \tilde{P}
$$

so $\tilde{P}$ is $V$-prime. As we observed above, since $T$ is Noetherian and $\operatorname{char} F=0$, this implies that $\tilde{P}$ is prime.

The above two results now combine to form the main theorem of this paper which at this point is essentially all notation and requires no additional proof.

THEOREM 4.3. Let $U=U(V, R, K)$ be given with $R$ prime and right Noetherian, $\operatorname{dim}_{K} V / R<\infty$ and $\operatorname{char} K=0$. Let $S=Q_{s}(R), F=\mathbf{Z}(S)$ and $U^{\prime}=$ $U\left(V^{\prime}, S, F\right) \supseteq U$ where $V^{\prime}=S+F V$. Then $T=\mathbf{C}_{U^{\prime}}(S)=U(W, F, F)$ is a twisted enveloping algebra of the finite-dimensional $F$-Lie algebra $W=\mathbf{C}_{V^{\prime}}(S)$ and $V$ acts on $T$ as derivations. Furthermore there exist one-to-one order preserving correspondences between the primes $P$ of $U$ with $P \cap R=0$, the primes $P^{\prime}$ of $U^{\prime}$ with $P^{\prime} \cap S=0$ and the $V$-stable primes $Q$ of $T$. Specifically these maps are given by

where

$$
Q \rightarrow Q U^{\prime}=P^{\prime}, \quad P^{\prime} \rightarrow P^{\prime} \cap U=P, \quad P \rightarrow \tilde{P}=Q
$$

$$
\tilde{P}=\{\alpha \in T \mid \text { there exists } 0 \neq A \triangleleft R \text { with } A \alpha \subseteq P\}
$$


We briefly consider several corollaries. Tho most obvious one concerns incomparability and prime length. As we observed earlier, if $U=U(V, R, K)$ with $\operatorname{dim}_{K} V / R=n$, then the associated graded ring of $U$ is isomorphic to the polynomial ring $R\left[x_{1}, x_{2}, \ldots, x_{n}\right]$ in $n$ variables. It follows from $[3, \S 3.5]$ that if $R$ is right Noetherian then

$$
\mathrm{K} \operatorname{dim} U \leq \mathrm{K} \operatorname{dim} R\left[x_{1}, x_{2}, \ldots, x_{n}\right] \leq \mathrm{K} \operatorname{dim} R+n .
$$

In particular since $U$ is also Noetherian, we have

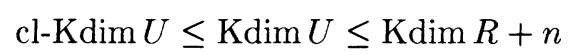

where the classical Krull dimension of a ring is its prime length. Among other things, the next result bounds $\operatorname{cl}-\mathrm{K} \operatorname{dim} U$ in terms of $n$ and $\operatorname{cl}-K \operatorname{dim} R$, although the bound is surely not sharp.

COROLlary 4.4. Let $U=U(V, R, K)$ be given with $R$ right Noetherian, $\operatorname{dim}_{K} V / R=n<\infty$ and $\operatorname{char} K=0$. If $P_{0}<P_{1}<\cdots<P_{n+1}$ are prime ideals of $U$, then $P_{0} \cap R<P_{n+1} \cap R$. Hence

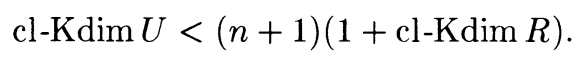

Proof. Since $P_{0}$ is a prime ideal of $U, P_{0} \cap R$ is a $V$-stable ideal of $R$ which is easily seen to be $V$-prime. Thus since $R$ is right Noetherian and char $K=0$, it follows that $P_{0} \cap R$ is prime. Now mod out by $\left(P_{0} \cap R\right) U \subseteq P_{0}$ using Corollary 1.8 to obtain

$$
\bar{U}=U /\left(P_{0} \cap R\right) U=U(\bar{V}, \bar{R}, K) .
$$

Then $\bar{U}$ contains the strictly increasing chain of primes $\bar{P}_{0}<\bar{P}_{1}<\cdots<\bar{P}_{n+1}$ and $\operatorname{dim}_{K} \bar{V} / \bar{R}=n$. Furthermore $\bar{P}_{0} \cap \bar{R}=0$ and $\bar{R}$ is prime. Thus replacing $U$ by $\bar{U}$ if necessary, it suffices to assume that $R$ is prime and $P_{0} \cap R=0$.

We apply Theorem 4.3 and its notation. Since $W \subseteq S+F V$ and $W \cap S=F$ we have $\operatorname{dim}_{F} W / F \leq \operatorname{dim}_{K} V / R=n$. Now suppose that $P_{n+1} \cap R=0$. Then $P_{i} \cap R=0$ for all $i$ and we obtain the strictly increasing chain $\tilde{P}_{0}<\tilde{P}_{1}<\cdots<\tilde{P}_{n+1}$ of $V$-stable primes of $T=U(W, F, F)$. But $F$ is a field so

$$
\operatorname{cl}-\mathrm{Kdim} U(W, F, F) \leq \mathrm{Kdim} F+n=n
$$

and we have a contradiction. We conclude therefore that $P_{n+1} \cap R>0=P_{0} \cap R$.

Finally if $P_{0}<P_{1}<\cdots<P_{m}$ is a strictly increasing chain of primes of $U$, then by the above

$$
P_{0} \cap R<P_{n+1} \cap R<P_{2(n+1)} \cap R<\cdots
$$

is a strictly increasing chain of primes of $R$. Since this chain can contain at most $1+\operatorname{cl}-\mathrm{Kdim} R$ primes, we conclude that $(n+1)(1+\mathrm{cl}-\mathrm{Kdim} R)>m$ as required.

Another corollary concerns the semiprimitivity of $U / P$. Again we assume that $R$ is prime, char $K=0$ and that $P$ is a prime ideal of $U=U(V, R, K)$ with $P \cap R=0$. Furthermore $R$ is right Noetherian and $\operatorname{dim}_{K} V / R<\infty$. We use the notation of Theorem 4.3. In particular, $U^{\prime}=U\left(V^{\prime}, S, F\right) \supseteq U, T=\mathbf{C}_{U^{\prime}}(S)$ and $\tilde{P}$ is the $V$-stable prime ideal of $T$ determined by $P$. Furthermore, by Theorem 2.2, $U^{\prime}=U\left(V^{\prime \prime}, S \otimes T, F\right)$ where

$$
V^{\prime \prime}=S \otimes_{F} T+V^{\prime}=S \otimes T+F V .
$$


LEMMA 4.5. Let $I \subseteq S \otimes T$.

(i) If $I$ is an $(R, R)$-subbimodule of $S \otimes T$ properly containing $S \otimes \tilde{P}$, then there exist $0 \neq A \triangleleft R$ and $\alpha \in T \backslash \tilde{P}$ with $A \alpha \subseteq I$.

(ii) If $I$ is an $(S \otimes T \cap U, S \otimes T \cap U)$-subbimodule of $S \otimes T$ not contained in $S \otimes \tilde{P}$, then $I$ contains a regular element modulo $S \otimes \tilde{P}$.

ProOF. (i) This is a standard fact (which does not require char $K=0$ ), and we obtain it easily from our earlier work. Observe that $S \otimes T=U(S \otimes T, S \otimes T, F)$ and that $T=\mathbf{C}_{S \otimes T}(S)$. Thus by Lemma $2.3, \bar{T}=\mathbf{C}_{S \otimes \bar{T}}(S)$ where $\bar{T}=T / \tilde{P}$. Furthermore $\bar{I}=I /(S \otimes \tilde{P})$ is a nonzero $(R, R)$-subbimodule of $U(S \otimes \bar{T}, S \otimes \bar{T}, F)$ so by Lemma 3.4 there exist $0 \neq A \triangleleft R$ and $0 \neq \bar{\alpha} \in \bar{T}$ with $A \bar{\alpha} \subseteq \bar{I}$. If $\alpha$ is any inverse image of $\bar{\alpha}$ in $T$, then $\alpha \in T \backslash \tilde{P}$ and $A \alpha \subseteq I$.

(ii) We will freely use the fact that any regular element of $S$ is regular in $S \otimes \bar{T}$. Let $I_{1}=T I T+S \otimes \tilde{P}$ so that $I_{1}$ is an $(R, R)$-subbimodule of $S \otimes T$ properly containing $S \otimes \tilde{P}$. Thus by (i) above there exist $0 \neq A \triangleleft R$ and $\alpha \in T \backslash \tilde{P}$ with $A \alpha \subseteq I_{1}$. Since $I_{1}$ is also a $(T, T)$-bimodule, it follows that $A \otimes B=A B \subseteq I_{1}$, where $B$ is the ideal of $T$ generated by $\alpha$ and $\tilde{P}$. Now $T / \tilde{P}$ is a prime Noetherian ring so $B$ contains an element $\beta$ regular modulo $\tilde{P}$. Furthermore $R$ is prime Noetherian so $A$ contains an element $c$ which is regular in $R$. But nonzero right and left ideals of $S$ meet $R$ nontrivially, so $c$ is also regular in $S$. It follows easily that $c \beta \in I_{1}$ is regular modulo $S \otimes \tilde{P}$. Finally

$$
c \beta \in I_{1}=T I T+S \otimes \tilde{P}
$$

so

$$
c \beta \equiv \sum_{i} t_{i} \eta_{i} t_{i}^{\prime} \bmod S \otimes \tilde{P}
$$

where $\eta_{i} \in I$ and $t_{i}, t_{i}^{\prime} \in T$. We can now choose $0 \neq D, D^{\prime} \triangleleft R$ with $D t_{i} \subseteq U$ and $D^{\prime} t_{i}^{\prime} \subseteq U$ for all $i$. Again $D$ and $D^{\prime}$ contain elements $d$ and $d^{\prime}$ respectively which are regular in $S$ so that

$$
d c \beta d^{\prime} \equiv \sum_{i}\left(d t_{i}\right) \eta_{i}\left(d^{\prime} t_{i}^{\prime}\right) \quad \bmod S \otimes \tilde{P}
$$

and the right-hand term is contained in

$$
(S \otimes T \cap U) I(S \otimes T \cap U) \subseteq I .
$$

Since $d$ and $d^{\prime}$ are also regular modulo $S \otimes \tilde{P}$, it follows that $d c \beta d^{\prime}$ is regular modulo $S \otimes \tilde{P}$. Thus $\sum_{i}\left(d t_{i}\right) \eta_{i}\left(d^{\prime} t_{i}^{\prime}\right) \in I$ is regular modulo $S \otimes \tilde{P}$.

Since $V^{\prime \prime}=S \otimes T+F V$, we can choose $x_{1}, x_{2}, \ldots, x_{m} \in V$ to be an $F$-basis for a complement for $S \otimes T$ in $V^{\prime \prime}$. Thus every element of $U^{\prime}$ is uniquely writable as $u^{\prime}=\sum_{\sigma} q_{\sigma} \sigma$ where $\sigma$ is a monic monomial in the $x$ 's and $q_{\sigma} \in S \otimes T$. As usual we will order the $\sigma$ 's first by degree and then lexicographically within terms of the same degree. If $\tau$ is the largest monomial in the support of $u^{\prime}$ then $\tau$ is the monomial degree of $u^{\prime}$ and $q_{\tau}$ is its leading coefficient.

LEMMA 4.6. (i) Let $u \in U$ and write $u=\sum_{\sigma} q_{\sigma} \sigma$ as above. Then $u \in P$ if and only if all $q_{\sigma} \in S \otimes \tilde{P}$. 
(ii) Let $J$ be an ideal of $U$ properly containing $P$. Then there exists $u \in J$ whose leading coefficient is regular modulo $S \otimes \tilde{P}$.

ProOF. (i) We apply Theorem 4.3 and set $P^{\prime}=P U^{\prime}=(S \otimes \tilde{P}) U^{\prime}$. If $u \in P$ then $u \in P^{\prime}$ so all $q_{\sigma} \in S \otimes \tilde{P}$. Conversely if all $q_{\sigma} \in S \otimes \tilde{P}$ then $u \in U \cap P^{\prime}=P$.

(ii) Choose $u \in J \backslash P$ of minimal monomial degree $\tau$ and say $u=\sum q_{\sigma} \sigma$. We show that $q_{\tau} \notin S \otimes \tilde{P}$. Suppose by way of contradiction that $q_{\tau} \in S \otimes \tilde{P}$ and choose $0 \neq B \triangleleft R$ with $B q_{\tau} \subseteq U$. As we observed earlier, $B$ contains an element $b$ which is regular in $S \otimes T$ modulo $S \otimes \tilde{P}$. We consider $b u=\sum_{\sigma} b q_{\sigma} \sigma \in J$. Note that $b q_{\tau} \in U \cap(S \otimes \tilde{P}) \subseteq P$ and hence $b q_{\tau} \tau \in P \subseteq J$ since $\tau \in U$. Thus $b u-b q_{\tau} \tau \in J$ and this element has smaller monomial degree. By definition of $\tau$ this implies that $b u-b q_{\tau} \tau \in P$ so $b u \in P$ and hence, by (i), $b q_{\sigma} \in S \otimes \tilde{P}$ for all $\sigma$. But $b$ is regular modulo $S \otimes \tilde{P}$ so we have $q_{\sigma} \in S \otimes \tilde{P}$ for all $\sigma$ and thus, by (i) again, $u \in P$, a contradiction. Thus $q_{\tau} \notin S \otimes \tilde{P}$.

With $\tau$ as above let

$$
I=\left\{q \in S \otimes T \mid \sum_{\sigma \leq \tau} a_{\sigma} \sigma \in J \text { with } a_{\sigma} \in S \otimes T \text { and } q=a_{\tau}\right\} .
$$

$I$ is clearly closed under addition and since $J \triangleleft U$ it follows immediately that $I$ is a left $(S \otimes T \cap U)$-submodule of $S \otimes T$. On the other hand, if $q \in S \otimes T$ then

$$
\sigma q=q \sigma+\text { lower degree terms }
$$

implies, since $\tau$ is the leading term, that $I$ is also a right $(S \otimes T \cap U)$-submodule. As we observed above, $q_{\tau} \in I$ and $q_{\tau} \notin S \otimes \tilde{P}$. Thus $I$ is an $(S \otimes T \cap U, S \otimes T \cap U)$ subbimodule of $S \otimes T$ not contained in $S \otimes \tilde{P}$. We conclude from Lemma 4.5(ii) that $I$ contains an element regular modulo $S \otimes \tilde{P}$. Thus there exists

$$
\sum_{\sigma \leq \tau} b_{\sigma} \sigma \in J
$$

with $b_{\sigma} \in S \otimes T$ and $b_{\tau}$ regular modulo $S \otimes \tilde{P}$. This completes the proof.

Let $\delta$ be a derivation of the prime ring $R$. Then $\delta$ is said to be X-inner on $R$ if its unique extension to $S=Q_{s}(R)$ is inner, that is if there exists $d \in S$ with $\delta(s)=[s, d]$ for all $s \in S$. We say that $V$ is $\mathrm{X}$-inner on $R$ if each $\delta_{v}$ is $\mathrm{X}$-inner. Continuing with the notation of Theorem 4.3 we have

LEMMA 4.7. $V$ is X-inner on $R$ if and only if $S+F V=V^{\prime}=S+W$.

Proof. If $V^{\prime}=S+W$, then $V \subseteq S+W$. Since $W$ centralizes $S$, it follows that each $v \in V$ acts on $S$ like an element of $S$, so $V$ is $\mathrm{X}$-inner. Conversely assume that $V$ is X-inner. If $v \in V$ then there exists $d \in S$ with $[s, v]=\delta_{v}(s)=[s, d]$ for all $s \in S$. Thus $v-d \in \mathbf{C}_{V^{\prime}}(S)=W$ and $v \in S+W$. This implies that $V \subseteq S+W$ and then clearly $V^{\prime}=S+F V \subseteq S+W$.

We can now obtain our result on semiprimitivity.

COROLlaRY 4.8. Let $U=U(V, R, K)$ be given with $R$ prime and right Noetherian, $\operatorname{dim}_{K} V / R<\infty$ and char $K=0$. Let $P$ be a prime ideal of $U$ with $P \cap R=0$. If $V$ is not $X$-inner on $R$, then $U / P$ is a semiprimitive ring. 
ProOF. We use the notation as developed above. In particular, since $V$ is not $\mathrm{X}$-inner on $R, S+W$ is properly smaller than $V^{\prime}$ and thus $S \otimes T$ is properly smaller than $V^{\prime \prime}$. This means that the set $\left\{x_{1}, x_{2}, \ldots, x_{m}\right\}$ is nonempty.

Let $J / P$ be the Jacobson radical of $U / P$ and assume by way of contradiction that $J>P$. Then by Lemma 4.6(ii), $J$ contains an element $\alpha=\sum_{\sigma \leq \tau} a_{\sigma} \sigma$ with $a_{\sigma} \in S \otimes T$ and $a_{\tau}$ regular modulo $S \otimes \tilde{P}$. If $\tau=1$ then we replace $\alpha$ by the element $\alpha x_{1} \in J$ which will have the same property. Thus we can assume that $\operatorname{deg} \tau>0$. Since $\alpha \in J, 1+\alpha$ is invertible modulo $P$ and we let $\beta=\sum_{\lambda} b_{\lambda} \lambda \in U$ with $b_{\lambda} \in S \otimes T$ and $(1+\alpha) \beta \equiv 1 \bmod P$. Certainly $\beta \notin P$ so by Lemma 4.5 (i) there exists $\mu$ with $b_{\mu} \notin S \otimes \tilde{P}$. Choose $\mu$ maximal with this property. Observe that if $\lambda>\mu$ then $b_{\lambda} \in S \otimes \tilde{P} \subseteq P^{\prime}$. Hence if $\gamma=\sum_{\lambda \leq \mu} b_{\lambda} \lambda$, then $(1+\alpha) \gamma \equiv 1$ $\bmod P^{\prime}$.

Now let $\rho$ be the monic monomial in the $x$ 's such that

$$
\tau \mu=\rho+\text { lower degree terms. }
$$

Since $\operatorname{deg} \tau \geq 1$, it follows that the coefficient of $\rho$ in $(1+\alpha) \gamma$ is precisely $a_{\tau} b_{\mu}$. Furthermore since $\operatorname{deg} \rho \geq \operatorname{deg} \tau \geq 1$ and $(1+\alpha) \gamma \equiv 1 \bmod P^{\prime}$, the coefficient of $\rho$ in this product is contained in $S \otimes \tilde{P}$. In other words, $a_{\tau} b_{\mu} \in S \otimes \tilde{P}$. But $a_{\tau}$ is regular modulo $S \otimes \tilde{P}$ and $b_{\mu} \notin S \otimes \tilde{P}$, so we have a contradiction. We conclude that $J=P$ as required.

We note that if $R$ is commutative, then $S$ is its field of fractions. Thus $\delta$ is an $\mathrm{X}$-inner derivation on $R$ if and only if $\delta=0$. With this observation we see that Corollary 4.8 extends [4, Theorem 4.1] in case char $K=0$.

5. The Poincaré-Birkhoff-Witt theorem. In this final section we prove Theorem 1.3, the PBW theorem. As will be apparent, the fact that we are not dealing with $K$-algebras makes the proof much more tedious. Furthermore, under these conditions it seems appropriate to use Witt's idea [8] of constructing the regular representation of $U$.

We are given $V \supseteq R \supseteq K$ where $K$ is a field, $R$ is an associative $K$-algebra with 1 and $V$ is a left $K$-vector space. Furthermore $V$ is a Lie ring under the operation [, ] and $V \supseteq R \supseteq K$ satisfies the conditions (L1)-(L3). In particular for all $v, w \in V$ and $a, b \in K$ we have

$$
[a v, b w]=a b[v, w]+a[v, b] w-b[w, a] v .
$$

Suppose that $b=1$ and $w=r \in R$. Since $V$ acts as derivations on $K$ and since $K$ is central in $R$, it follows that $[v, b]=[v, 1]=0$ and $[w, a]=[r, a]=0$. Thus we obtain $[a v, r]=a[v, r]$ for all $a \in K, v \in V$ and $r \in R$. We will use this freely.

Let $x_{1}, x_{2}, \ldots$ be a well-ordered $K$-basis for a complement for $R$ in $V$. We begin by forming the ring $\mp$ which is freely generated by $R$ and the symbols $x_{1}, x_{2}, \ldots$ Then $R+\sum_{i} K x_{i}$ is a direct sum in $\mathcal{\exists}$ and in this way we view $\mathcal{F} \supseteq V$. In fact $\mathcal{F}$ is then generated by $V$ and we let [,] denote the given Lie bracket for $V$.

Let $M$ be the free left $R$-module with $R$-basis consisting of the formal monic monomials

$$
\mu=X_{i_{1}} X_{i_{2}} \cdots X_{i_{k}}, \quad i_{1} \leq i_{2} \leq \cdots \leq i_{k}
$$

where each $X_{i}$ corresponds to an $x_{i}$. We deal with these monomials in the usual multiplicative way and in particular we write the empty product as 1 . The degree 
of $\mu$ is $\operatorname{deg} \mu=k$ and for $\sum r_{j} \mu_{j} \in M$ we set

$$
\operatorname{deg}\left(\sum r_{j} \mu_{j}\right)=\max \left\{\operatorname{deg} \mu \mid r_{j} \neq 0\right\} \text {. }
$$

For each integer $n$ set $M_{n}=\{\alpha \in M \mid \operatorname{deg} \alpha \leq n\}$ and let $E=\operatorname{End}(M)$ be the endomorphism ring of $M$ as an abelian group.

Since $M$ is a free left $R$-module, there is a ring monomorphism ${ }^{*}: R \rightarrow E$ given by $r^{*}(s \mu)=(r s) \mu$ for $r, s \in R$. In addition, for each $i$ we define $x_{i}^{*} \in E$ linearly by the formulas:

$$
\begin{gathered}
x_{i}^{*} 1=X_{i}, \\
x_{i}^{*}\left(X_{j} \lambda\right)= \begin{cases}X_{i} X_{j} \lambda & \text { if } i \leq j, \\
x_{j}^{*} x_{i}^{*} \lambda+\left[x_{i}, x_{j}\right]^{*} \lambda & \text { if } i>j,\end{cases} \\
x_{i}^{*}(r \mu)=r x_{i}^{*} \mu+\left[x_{i}, r\right] \mu .
\end{gathered}
$$

Here $X_{j} \lambda$ is a monic monomial starting with an $X_{j}$ factor, $\mu$ is any monic monomial and $r \in R$.

LEMMA 5.1. * yields a well-defined ring homomorphism from $₹$ to E. Furthermore for any $r \in R, r^{*} x_{i}^{*} M_{n} \subseteq M_{n+1}$.

ProOF. We have to show that (M1)-(M3) uniquely define each $x_{i}^{*} \in E$. Indeed we show by induction on $n$ that each $x_{i}^{*}$ is uniquely defined on $M_{n}$ and that $r^{*} x_{i}^{*} M_{n} \subseteq M_{n+1}$. If $n=0$ this is immediate from (M1) and (M3). Now suppose the result holds for $n$. Since (M3) is linear in $r$, we need only check that, for each monic monomial $\mu$ of degree $n+1, x_{i}^{*} \mu$ is defined and has the appropriate degree.

We proceed by induction on the well-ordered subscripts. Write $\mu=X_{j} \lambda$. If $i \leq j$ (in particular if $i=1$ ) then

$$
x_{i}^{*} \mu=x_{i}^{*} X_{j} \lambda=X_{i} X_{j} \lambda
$$

has the desired property. On the other hand, if $j<i$ then since $\lambda \in M_{n}, x_{i}^{*} \lambda$ and $\left[x_{i}, x_{j}\right]^{*} \lambda$ are both defined and are contained in $M_{n+1}$. Note that $\left[x_{i}, x_{j}\right]$ is uniquely writable as $\left[x_{i}, x_{j}\right]=r+\sum a_{k} x_{k}$ with $r \in R$ and $a_{k} \in K$ and then

$$
\left[x_{i}, x_{j}\right]^{*} \lambda=r^{*} \lambda+\sum_{k} a_{k}^{*} x_{k}^{*} \lambda .
$$

By induction $x_{j}^{*}\left(x_{i}^{*} \lambda\right)$ is also defined and contained in $M_{n+2}$. Equation (M2) therefore yields $x_{i}^{*} \mu$ in this case also.

Thus each $x_{i}^{*} \in E$ and since $\mathcal{F}$ is freely generated by $R$ and the $x_{i},{ }^{*}$ extends to a ring homomorphism ${ }^{*}: \mathcal{F} \rightarrow E$.

The goal is to understand the kernel of $*$. We start with

LEMMA 5.2. For all $r \in R, a \in K$ and $x_{i}$ we have

$$
\left(a x_{i}\right)^{*} r^{*}=r^{*}\left(a x_{i}\right)^{*}+\left[a x_{i}, r\right]^{*} .
$$

PROOF. Since $\left[a x_{i}, r\right]=a\left[x_{i}, r\right]$, it clearly suffices to assume $a=1$. Since the map $r \rightarrow\left[x_{i}, r\right]$ is a derivation on $R$, (M3) yields

$$
\begin{aligned}
\left(r^{*} x_{i}^{*}+\left[x_{i}, r\right]^{*}\right) s \mu & =r s x_{i}^{*} \mu+\left(r\left[x_{i}, s\right]+\left[x_{i}, r\right] s\right) \mu \\
& =r s x_{i}^{*} \mu+\left[x_{i}, r s\right] \mu=x_{i}^{*} r^{*} s \mu .
\end{aligned}
$$


LEMMA 5.3. Let $n$ be fixed and suppose that $x_{i}^{*} x_{j}^{*}$ and $x_{j}^{*} x_{i}^{*}+\left[x_{i}, x_{j}\right]^{*}$ agree in their action on all monic monomials of degree $\leq n$. Then

$$
x_{i}^{*} x_{j}^{*}=x_{j}^{*} x_{i}^{*}+\left[x_{i}, x_{j}\right]^{*} \text { on } M_{n} .
$$

PROOF. If $r \mu \in M_{n}$, then two applications of Lemma 5.2 yield

$$
\begin{aligned}
x_{i}^{*} x_{j}^{*} r \mu & =x_{i}^{*} x_{j}^{*} r^{*} \mu=x_{i}^{*}\left(r^{*} x_{j}^{*}+\left[x_{j}, r\right]\right) \mu \\
& =\left(r^{*} x_{i}^{*}+\left[x_{i}, r\right]\right) x_{j}^{*} \mu+x_{i}^{*}\left[x_{j}, r\right] \mu \\
& =\left(r x_{i}^{*} x_{j}^{*}+\left[x_{i}, r\right] x_{j}^{*}+x_{i}^{*}\left[x_{j}, r\right]\right) \mu .
\end{aligned}
$$

Hence by subtracting the analogous formula with $i$ and $j$ interchanged, we have

$$
\begin{aligned}
\left(x_{i}^{*} x_{j}^{*}-x_{j}^{*} x_{i}^{*}\right) r \mu= & r\left(x_{i}^{*} x_{j}^{*}-x_{j}^{*} x_{i}^{*}\right) \mu+\left(\left[x_{i}, r\right] x_{j}^{*}-x_{j}^{*}\left[x_{i}, r\right]\right) \mu \\
& +\left(x_{i}^{*}\left[x_{j}, r\right]-\left[x_{j}, r\right] x_{i}^{*}\right) \mu .
\end{aligned}
$$

Since $\left(x_{i}^{*} x_{j}^{*}-x_{j}^{*} x_{i}^{*}\right) \mu=\left[x_{i}, x_{j}\right]^{*} \mu$ by assumption and since $\left[x_{i}, r\right],\left[x_{j}, r\right] \in R$, Lemma 5.2 yields

$$
\left(x_{i}^{*} x_{j}^{*}-x_{j}^{*} x_{i}^{*}\right) r \mu=r\left[x_{i}, x_{j}\right]^{*} \mu+\left(\left[\left[x_{i}, r\right], x_{j}\right]+\left[x_{i},\left[x_{j}, r\right]\right]\right) \mu .
$$

Finally the Jacobi identity applies to the second term on the above right and we obtain

$$
\left(x_{i}^{*} x_{j}^{*}-x_{j}^{*} x_{i}^{*}\right) r \mu=\left(r\left[x_{i}, x_{j}\right]^{*}+\left[\left[x_{i}, x_{j}\right], r\right]\right) \mu=\left[x_{i}, x_{j}\right]^{*} r \mu
$$

where the latter follows from (M3) and $K$-linearity.

Notice that the previous two lemmas arise because of the presence of $R$ and a noncentral $K$. The same is true for the first half of the next result.

LEMMA 5.4. Let $n$ be fixed and suppose that for all $i, j$

$$
x_{i}^{*} x_{j}^{*}=x_{j}^{*} x_{i}^{*}+\left[x_{i}, x_{j}\right]^{*} \text { on } M_{n} .
$$

Then for all $\alpha, \beta \in V$ we have

$$
\alpha^{*} \beta^{*}=\beta^{*} \alpha^{*}+[\alpha, \beta]^{*} \text { on } M_{n} .
$$

Furthermore for all $i, j, k$

$$
\begin{aligned}
& x_{k}^{*}\left[x_{i}, x_{j}\right]^{*}+\left[x_{i}, x_{k}\right]^{*} x_{j}^{*}+x_{i}^{*}\left[x_{j}, x_{k}\right]^{*} \\
& \quad=\left[x_{i}, x_{j}\right]^{*} x_{k}^{*}+x_{j}^{*}\left[x_{i}, x_{k}\right]^{*}+\left[x_{j}, x_{k}\right]^{*} x_{i}^{*} \text { on } M_{n} .
\end{aligned}
$$

PROOF. For the first part, by linearity it suffices to show that for all $r, s \in R$, $a, b \in K$ and $x_{i}, x_{j}$

$$
\begin{gathered}
r^{*} s^{*}-s^{*} r^{*}=[r, s]^{*} \quad \text { on } M_{n} \\
\left(a x_{i}\right)^{*} r^{*}-r^{*}\left(a x_{i}\right)^{*}=\left[a x_{i}, r\right]^{*} \text { on } M_{n}
\end{gathered}
$$

and

$$
\left(a x_{i}\right)^{*}\left(b x_{j}\right)^{*}-\left(b x_{j}\right)^{*}\left(a x_{i}\right)^{*}=\left[a x_{i}, b x_{j}\right]^{*} \text { on } M_{n} .
$$

The first is clear since ${ }^{*}$ is a ring homomorphism and $[r, s]=r s-s r$ by (L3). The second is just Lemma 5.2 .

For the third, we note from Lemma 5.2 that

$$
\left(a x_{i}\right)^{*}\left(b x_{j}\right)^{*}=a\left(b x_{i}^{*}+\left[x_{i}, b\right]\right) x_{j}^{*}=a b x_{i}^{*} x_{j}^{*}+a\left[x_{i}, b\right] x_{j}^{*} .
$$


Hence by subtracting the analogous formula with $i$ and $j$ interchanged we have

$$
\left(a x_{i}\right)^{*}\left(b x_{j}\right)^{*}-\left(b x_{j}\right)^{*}\left(a x_{i}\right)^{*}=a b\left(x_{i}^{*} x_{j}^{*}-x_{j}^{*} x_{i}^{*}\right)+a\left[x_{i}, b\right] x_{j}^{*}-b\left[x_{j}, a\right] x_{i}^{*} .
$$

Since $x_{i}^{*} x_{j}^{*}-x_{j}^{*} x_{i}^{*}=\left[x_{i}, x_{j}\right]^{*}$ on $M_{n}$, the result follows from the formula for $\left[a x_{i}, b x_{j}\right]$ in (L3).

For the second part, let $\tau$ denote the left-hand side minus the right-hand side. Then by the first part, the action of $\tau$ on $M_{n}$ is given by

$$
\tau=\left[x_{k},\left[x_{i}, x_{j}\right]\right]^{*}+\left[\left[x_{i}, x_{k}\right], x_{j}\right]^{*}+\left[x_{i},\left[x_{j}, x_{k}\right]\right]^{*} .
$$

Since $\left[\left[x_{i}, x_{k}\right], x_{j}\right]=\left[x_{j},\left[x_{k}, x_{i}\right]\right]$, it follows from the Jacobi identity that $\tau$ is zero on $M_{n}$.

Now we come to the key ingredient.

LEMMA 5.5. For all $\alpha, \beta \in V$

$$
\alpha^{*} \beta^{*}=\beta^{*} \alpha^{*}+[\alpha, \beta]^{*} .
$$

ProOF. We show by induction on $n$ that

$$
\alpha^{*} \beta^{*}=\beta^{*} \alpha^{*}+[\alpha, \beta]^{*} \quad \text { on } M_{n} .
$$

In view of Lemmas 5.3 and 5.4 , it suffices to check that $x_{i}^{*} x_{j}^{*}$ and $x_{j}^{*} x_{i}^{*}+\left[x_{i}, x_{j}\right]^{*}$ agree on all monic monomials of degree $\leq n$. Furthermore by symmetry we can assume that $i>j$. The $n=0$ case is immediate from (M1) and (M2).

Assume that the result holds for $n$. We order the pairs of subscripts $(i, j)$ with $(i>j)$ lexicographically so that $(i, j) \geq\left(i^{\prime}, j^{\prime}\right)$ if and only if $i>i^{\prime}$ or $i=i^{\prime}$ and $j \geq j^{\prime}$. This is certainly a well-ordering and we show by induction on $(i, j)$ that

$$
x_{i}^{*} x_{j}^{*}=x_{j}^{*} x_{i}^{*}+\left[x_{i}, x_{j}\right]^{*} \text { on } M_{n+1} .
$$

Assume the above statement is true for all $\left(i^{\prime}, j^{\prime}\right)<(i, j)$.

Let $\mu=X_{k} \lambda$ be a monic monomial of degree $n+1$. There are two cases to consider according to whether $j>k$ or $j \leq k$. Suppose first that $j \leq k$. (In particular this occurs at the start of the induction where $(i, j)=(2,1)$.) Then by (M2)

Hence since $i>j$, (M2) yields

$$
x_{i}^{*} x_{j}^{*} \mu=x_{i}^{*} x_{j}^{*} X_{k} \lambda=x_{i}^{*} X_{j} X_{k} \lambda .
$$

$$
x_{i}^{*} x_{j}^{*} \mu=\left(x_{j}^{*} x_{i}^{*}+\left[x_{i}, x_{j}\right]^{*}\right) X_{k} \lambda=\left(x_{j}^{*} x_{i}^{*}+\left[x_{i}, x_{j}\right]^{*}\right) \mu
$$

as required.

On the other hand, if $j>k$ then by (M2)

$$
x_{i}^{*} x_{j}^{*} \mu=x_{i}^{*} x_{j}^{*} X_{k} \lambda=x_{i}^{*}\left(x_{k}^{*} x_{j}^{*}+\left[x_{j}, x_{k}\right]^{*}\right) \lambda .
$$

Thus since $(i, k)<(i, j)$ and $x_{j}^{*} \lambda \in M_{n+1}$, induction implies that

$$
x_{i}^{*} x_{j}^{*} \mu=\left(x_{k}^{*} x_{i}^{*}+\left[x_{i}, x_{k}\right]^{*}\right) x_{j}^{*} \lambda+x_{i}^{*}\left[x_{j}, x_{k}\right]^{*} \lambda .
$$

Furthermore since $\lambda \in M_{n}$ we have $x_{i}^{*} x_{j}^{*} \lambda=\left(x_{j}^{*} x_{i}^{*}+\left[x_{i}, x_{j}\right]^{*}\right) \lambda$ and hence

$$
x_{i}^{*} x_{j}^{*} \mu=\left(x_{k}^{*} x_{j}^{*} x_{i}^{*}+x_{k}^{*}\left[x_{i}, x_{j}\right]^{*}+\left[x_{i}, x_{k}\right]^{*} x_{j}^{*}+x_{i}^{*}\left[x_{j}, x_{k}\right]^{*}\right) \lambda .
$$

Again $\lambda \in M_{n}$ so the second part of Lemma 5.4 applies to yield

$$
x_{i}^{*} x_{j}^{*} \mu=\left(x_{k}^{*} x_{j}^{*} x_{i}^{*}+\left[x_{i}, x_{j}\right]^{*} x_{k}^{*}+x_{j}^{*}\left[x_{i}, x_{k}\right]^{*}+\left[x_{j}, x_{k}\right]^{*} x_{i}^{*}\right) \lambda .
$$


We now put this back together. Since $x_{i}^{*} \lambda \in M_{n+1}$ and $(j, k)<(i, j)$ we have

$$
\left(x_{k}^{*} x_{j}^{*}+\left[x_{j}, x_{k}\right]^{*}\right) x_{i}^{*} \lambda=x_{j}^{*} x_{k}^{*} x_{i}^{*} \lambda
$$

so

$$
x_{i}^{*} x_{j}^{*} \mu=\left(x_{j}^{*} x_{k}^{*} x_{i}^{*}+\left[x_{i}, x_{j}\right]^{*} x_{k}^{*}+x_{j}^{*}\left[x_{i}, x_{k}\right]^{*}\right) \lambda .
$$

Next since $\left(x_{k}^{*} x_{i}^{*}+\left[x_{i}, x_{k}\right]^{*}\right) \lambda=x_{i}^{*} x_{k}^{*} \lambda$, the expression simplifies to

$$
x_{i}^{*} x_{j}^{*} \mu=\left(x_{j}^{*} x_{i}^{*}+\left[x_{i}, x_{j}\right]^{*}\right) x_{k}^{*} \lambda .
$$

Finally since $\mu=X_{k} \lambda, k$ is smaller than or equal to the first subscript in $\lambda$ so $x_{k}^{*} \lambda=X_{k} \lambda=\mu$. In other words, we have shown that

$$
x_{i}^{*} x_{j}^{*} \mu=\left(x_{j}^{*} x_{i}^{*}+\left[x_{i}, x_{j}\right]^{*}\right) \mu
$$

as required.

We conclude that $x_{i}^{*} x_{j}^{*}$ and $\left(x_{j}^{*} x_{i}^{*}+\left[x_{i}, x_{j}\right]^{*}\right)$ agree on $M_{n}$ and on all monic monomials of degree $n+1$. Thus Lemma 5.3 yields

$$
x_{i}^{*} x_{j}^{*}=x_{j}^{*} x_{i}^{*}+\left[x_{i}, x_{j}\right]^{*} \text { on } M_{n+1} .
$$

By induction we deduce that the above holds for all $i>j$ and therefore by Lemma $5.4 \alpha^{*} \beta^{*}=\beta^{*} \alpha^{*}+[\alpha, \beta]^{*}$ on $M_{n+1}$ for all $\alpha, \beta \in V$. The result therefore follows by induction on $n$.

It is now a simple matter to prove the PBW theorem.

PROOF OF THEOREM 1.3. We consider the products $x_{i_{1}} x_{x_{2}} \cdots x_{i_{j}} \in \mathcal{F}$ with $i_{1} \leq i_{2} \leq \cdots \leq i_{j}$ and also call these monic monomials. Let $I$ be the two-sided ideal of $\bar{\xi}$ generated by all $\alpha \beta-\beta \alpha-[\alpha, \beta]$ with $\alpha, \beta \in V$. Thus for all $i, j$ and $r \in R$

$$
\begin{gathered}
x_{i} x_{j}-x_{j} x_{i} \equiv\left[x_{i}, x_{j}\right] \quad \bmod I . \\
x_{i} r-r x_{i} \equiv\left[x_{i}, r\right] \quad \bmod I .
\end{gathered}
$$

It follows from Lemma 1.2 that every element of $\mathcal{F}$ is congruent modulo $I$ to a finite sum $\sum r_{\mu} \mu$ where each $\mu$ is a monic monomial and $r_{\mu} \in R$.

Now we observe that the monic monomials are $R$-linearly independent modulo $I$. Indeed suppose $\sum r_{\mu} \mu \in I$ and consider the homomorphism * from $\mathcal{F}$ to $E$. By Lemma 5.5, I is in the kernel of * and thus $\sum r_{\mu}^{*} \mu^{*}=0$. In particular $\left(\sum r_{\mu}^{*} \mu^{*}\right) 1=$ 0 . But since $\mu=x_{i_{1}} x_{i_{2}} \cdots x_{i_{j}}$ is a product written in its natural order, it follows from (M1) and (M2) that

$$
\mu^{*} 1=\left(x_{i_{1}} x_{i_{2}} \cdots x_{i_{j}}\right)^{*} 1=X_{i_{1}} X_{i_{2}} \cdots X_{i_{j}} .
$$

In other words, each $\mu^{*} 1$ is a distinct monic monomial in $M$ and recall that $M$ is a free left $R$-module with these monomials as a basis. Hence $\sum r_{\mu}\left(\mu^{*} 1\right)=0$ implies that all $r_{\mu}=0$ as required.

Set $\mathcal{F} / I=U$. Then we have shown that the monic monomials $\mu+I \in U$ form a free left $R$-basis for $U$. In particular since $V=R \oplus \sum_{i} K x_{i}, V$ embeds isomorphically in $U$. Via this isomorphism we will view $V$ as being contained in $U$ and then $V$ clearly generates $U$. Furthermore by definition of $I$ we have $\alpha \beta-\beta \alpha=[\alpha, \beta]$ for all $\alpha, \beta \in V \subseteq U$. Thus $U$ has the appropriate structure for $U(V, R, K)$ as described in (U1), (U2) and Theorem 1.3. It remains to verify (U3).

To this end, let $W \supseteq S \supseteq K$ be given and let $T$ be a ring containing $W$ for which [, ] on $W$ is the restriction of the usual Lie bracket on $T$. Furthermore let 
- $: V \rightarrow W$ be a $K$-linear Lie homomorphism whose restriction to $R$ is a $K$-algebra homomorphism ${ }^{-}: R \rightarrow S$. Since $\mathcal{F}$ is freely generated by $R$ and the $x_{i}$, there exists a homomorphism $\sim: \mathcal{F} \rightarrow T$ with $\tilde{r}=\bar{r}$ and $\tilde{x}_{i}=\bar{x}_{i}$ for all $i$ and $r \in R$. By $K$-linearity we have $\tilde{v}=\bar{v}$ for all $v \in V \subseteq \mathcal{F}$. Now observe that for all $\alpha, \beta \in V \subseteq \mathcal{F}$,

$$
(\alpha \beta-\beta \alpha-[\alpha, \beta])^{\sim}=\bar{\alpha} \bar{\beta}-\bar{\beta} \bar{\alpha}-[\alpha, \beta]^{-}=\bar{\alpha} \bar{\beta}-\bar{\beta} \bar{\alpha}-[\bar{\alpha}, \bar{\beta}]
$$

since $^{-}$is a Lie homomorphism. Thus since [ , ] on $W$ is the restriction of the ring bracket on $T$, we see that $\alpha \beta-\beta \alpha-[\alpha, \beta] \in \operatorname{ker}(\sim)$. It follows that $\operatorname{ker}(\sim) \supseteq I$ so $\sim$ factors through $U=\mathcal{F} / I$ and the theorem is proved.

ADDED IN PROOF. Professor L. A. Bokut' kindly informed me of an announcement by U. A. Drozd and Ya. Halluf entitled Generalization of a theorem of Ado in the 18th Algebraic Conference, I, Kishinev, 1985, p. 175. Their expanded paper (in preparation as of September 1986) apparently contains a construction similar to the universal enveloping rings considered here.

\section{REFERENCES}

1. J. Bergen and S. Montgomery, Smash products and outer derivations, Israel J. Math. 53 (1986), 321-345.

2. W. Chin, Prime ideals in differential operator rings and crossed products of infinite groups, J. Algebra 106 (1987), 78-104.

3. J. Dixmier, Enveloping algebras, North-Holland, Amsterdam, 1977.

4. K. R. Goodearl and R. B. Warfield, Primitivity in differential operator rings, Math. Z. 180 (1982), 503-523.

5. N. Jacobson, Lie algebras, Wiley-Interscience, New York, 1962.

6. M. Lorenz and D. S. Passman, Prime ideals in crossed products of finite groups, Israel J. Math. 33 (1979), 89-132.

7. D. S. Passman, Computing the symmetric ring of quotients, J. Algebra 105 (1987), 207-235.

8. E. Witt, Treue Darstellung Liescher Ringe, J. Reine Angew. Math. 177 (1937), 152-160.

Department of Mathematics, University of Wisconsin-Madison, Madison, WISCONSIN 53706 